\title{
Theory of an Accurate Intermediary Orbit for Satellite Astronomy
}

\author{
John P. Vinti
}

(May 16, 1961)

\begin{abstract}
This paper derives an accurate intermediary orbit of an artificial satellite of an oblate planet. The drag-free motion takes place under the action of a gravitational potential which fits the even zonal harmoniss exactly through the second and approximately through the fourth, in the case of the earth. This potential leads to separability of the HamiltonJacobi equation.

Two alternative sets of orbital elements are set forth. The first set is related directly to initial conditions, but requires numerical factoring of a certain quartic to evaluate some of the integrals. The second set, on the other hand, permits exact factoring of both quartics that appear, but is not related directly to initial conditions, so that its members can best be obtained by a least-square fit of the solution over many orbital revolutions.

The final solution is given in terms of certain uniformizing variables, whose periodic terms are correct through the second order in the oblateness parameter and whose secular terms are exact, for the intermediary orbit. These exact solutions for the secular terms are expressed by means of certain rapidly converging series, with complete avoidance of elliptic integrals of the third kind. Sections 9 and 10 give a summary and a list of symbols.
\end{abstract}

\section{Introduction}

The author has introduced a gravitational potential $[1]^{2}$ for an axially symmetric planet which accounts rather accurately for its oblateness and yet still leads to separability of the problem of satellite motion. The resulting solution is expected to yield an "intermediary orbit" somewhat more accurate than those heretofore used, in that it accounts for all of the second zonal harmonic and for more than half of the fourth zonal harmonic. (Previous intermediary orbits $[2,3]$ have accounted for only part of the second harmonic and have neglected the fourth harmonic.) The residual fourth harmonic, the odd harmonics, the tesseral harmonics, the lunar-solar forces, and aerodynamic and electromagnetic drag are then to be considered as producing perturbations of this intermediary orbit.

Izsak [4] has already given an analytical solution for this intermediary orbit, with both periodic and secular terms correct through the second order in a certain oblateness parameter. His solution makes rather heavy demands on the reader's knowledge of linear fractional transformations and the theory of elliptic functions in the complex plane. The present paper avoids these complications, with elliptic integrals occurring only in the simple forms of the complete first and second kinds. Furthermore the resulting solution not only gives the periodic terms correctly to the second order, but gives the secular terms "exactly"; i.e., to arbitrarily high order. I wish to acknowledge very explicitly, however, that I am greatly indebted to Tzsak for the introduction of one of the sets of orbital elements that I use. Knowledge of this set, which permits exact factoring of a certain refractory quartic, has influenced my treatment of the whole problem.

\section{Statement of Problem}

If $\rho, \eta, \phi$ are the oblate spheroidal coordinates introduced in [1] and if $r, \theta, \phi$ and $X, Y, Z$ are the corresponding spherical and rectangular coordinates, then

$$
\begin{gathered}
X+i Y=r \cos \theta \exp i \phi=\left[\left(\rho^{2}+c^{2}\right)\left(1-\eta^{2}\right)\right]^{\frac{1}{2}} \exp i \phi, \\
Z=r \sin \theta=\rho \eta, \quad(-1 \leqq \eta \leqq 1) .
\end{gathered}
$$

\footnotetext{
1 This work was supported by the U.S. Air Force, through the Office of Scientific Research of the Air Research and Development Command.

${ }^{2}$ Figures in brackets indicate the literature references at the end of the paper.
} 
Here $r$ is the geocentric distance to the satellite and $\theta$ and $\phi$ are respectively its geocentric declination and right ascension. For sufficiently large $r, \rho \approx r$ and $\eta \approx \sin \theta$.

With the origin taken at the planet's center of mass, the intermediary orbit is then the path of a particle in the approximate potential field

$$
V_{a}=-\mu \rho\left(\rho^{2}+c^{2} \eta^{2}\right)^{-1},
$$

where $\mu$ is the product of the gravitational constant and the planetary mass and where

$$
c^{2}=r_{e}^{2} J_{2}
$$

Here $r_{e}$ is the equatorial radius of the planet and $J_{2}$ is the coefficient of the second zonal harmonic in the expansion of the planet's true potential in spherical harmonics. For the earth $J_{2}=(1.08)$ $10^{-3}$, to three significant figures.

According to [1], the coordinates $\rho, \eta, \phi$ satisfy the following equations, involving quadratures:

$$
\begin{aligned}
t+\beta_{1} & = \pm \int_{\rho_{1}}^{\rho} \rho^{2} F^{-\frac{1}{2}}(\rho) d \rho \pm c^{2} \int_{0}^{\eta} \eta^{2} G^{-\frac{1}{2}}(\eta) d \eta \\
\beta_{2} & =\mp \alpha_{2} \int_{\rho_{1}}^{\rho} F^{-\frac{1}{2}}(\rho) d \rho \pm \alpha_{2} \int_{0}^{\eta} G^{-\frac{1}{2}}(\eta) d \eta \\
\phi-\beta_{3} & =\mp c^{2} \alpha_{3} \int_{\rho_{1}}^{\rho}\left(\rho^{2}+c^{2}\right)^{-1} F^{-\frac{1}{2}}(\rho) d \rho \pm \alpha_{3} \int_{0}^{\eta}\left(1-\eta^{2}\right)^{-1} G^{-\frac{1}{2}}(\eta) d \eta .
\end{aligned}
$$

Here $F(\rho)$ and $G(\eta)$ are the quartic polynomials

$$
\begin{aligned}
& F(\rho)=c^{2} \alpha_{3}^{2}+\left(\rho^{2}+c^{2}\right)\left(-\alpha_{2}^{2}+2 \mu \rho+2 \alpha_{1} \rho^{2}\right), \\
& G(\eta)=-\alpha_{3}^{2}+\left(1-\eta^{2}\right)\left(\alpha_{2}^{2}+2 \alpha_{1} c^{2} \eta^{2}\right) .
\end{aligned}
$$

The $\alpha$ 's and $\beta$ 's are the Jacobi constants, with the energy $\alpha_{1}<0$ for satellite motion and with the polar component of angular momentum $\alpha_{3} \geq 0$ according as the orbit is direct or retrograde. To orient oneself, note that in the limiting case $c \rightarrow 0$ of Keplerian motion the separation constant $\alpha_{2}$ reduces to the total angular momentum, $-\beta_{1}$ to the time of passage through perigee, $\beta_{2}$ to the argument $\omega$ of perigee, and $\beta_{3}$ to the right ascension $\Omega$ of the ascending node. The Jacobi constants may be determined, at least in principle, from the initial conditions; we have more to say about this point later. The constant $\rho_{1}$ is the next-to-the-largest real zero of $F(\rho)$ and thus is that zero of $F(\rho)$ which is closest to the smaller zero of

$$
f(\rho) \equiv-\alpha_{2}^{2}+2 \mu \rho+2 \alpha_{1} \rho^{2} .
$$

To solve (2.5) through (2.9) for $\rho, \eta$, and $\phi$ as functions of $t$, we must first solve (2.5) and (2.6) for $\rho$ and $\eta$ and then substitute the results $\rho(t)$ and $\eta(t)$ into $(2.7)$ to determine $\phi(t)$. To do so we must first evaluate the above six integrals, which we shall obtain in terms of certain uniformizing variables. In turn, evaluating these integrals presupposes knowing how to factor the quartics $F(\rho)$ and $G(\eta)$.

\section{Factoring the Quartics: Orbital Elements $a_{0}, e_{0}, i_{0}, \beta_{1}, \beta_{2}, \beta_{3}$}

In the case of elliptic motion $(c=0)$ the perigee and apogee radii $r_{1}$ and $r_{2}$ would be the two zeros of $f(\rho)$, viz,

$$
\begin{aligned}
& r_{1}=a_{0}\left(1-e_{0}\right), \\
& r_{2}=a_{0}\left(1+e_{0}\right),
\end{aligned}
$$


where

$$
\begin{aligned}
& a_{\supset} \equiv-\frac{1}{2} \mu \alpha_{1}^{-1} \\
& e_{0}^{2} \equiv 1+2 \alpha_{1} \alpha_{2}^{2} \mu^{-2} .
\end{aligned}
$$

Jn our present problem, with $c \neq 0$, we may still define constants $a_{0}$ and $e_{0}$ by (3.3) and (3.4), as well as another constant

$$
i_{0} \equiv \cos ^{-1}\left(\alpha_{3} / \alpha_{2}\right) \text {. }
$$

The constants $a_{0}, e_{0}, i_{0}, \beta_{1}, \beta_{2}$, and $\beta_{3}$ constitute one possible set of orbital constants. We may also introduce the corresponding semi-latus rectum $p_{0}$, defined by

so that

$$
p_{0} \equiv a_{0}\left(1-e_{0}^{2}\right) \text {, }
$$

$$
\alpha_{2}^{2}=\mu p_{0}
$$

A determination of $\alpha_{1}, \alpha_{2}$, and $\alpha_{3}$ would then furnish $a_{0}, e_{0}$, and $i_{0}$. If the subscript $i$ denotes initial values, then (per unit mass)

$$
\begin{aligned}
& \alpha_{1}=\frac{1}{2} u_{i}^{2}-\mu \rho_{i}\left(\rho_{i}^{2}+c^{2} \eta_{i}^{2}\right)^{-1}, \\
& \alpha_{3}=r_{i}^{2} \cos ^{2} \theta_{i} \dot{\phi}_{i}=X_{i} \dot{Y}_{i}-Y_{i} \dot{X}_{i},
\end{aligned}
$$

where $u$ means speed and a superscript dot denotes the time derivative. Also, by equations (50), (59.1), (13.2), (10.2), and (55) of [1],

$$
\alpha_{2}^{2}=\left(1-\eta_{i}^{2}\right)^{-1}\left[\left(\rho_{i}^{2}+c^{2} \eta_{i}^{2}\right)^{2} \dot{\eta}_{i}^{2}+\alpha_{3}^{2}-2 \alpha_{1} c^{2} \eta_{i}^{2}\left(1-\eta_{i}^{2}\right)\right] .
$$

Thus a knowledge of the initial coordinates and their initial derivatives would determine the $\alpha$ 's and thus the constants $a_{0}, e_{0}$, and $i_{0}$.

A knowledge of their numerical values would then permit a numerical solution of the quartic equation $F(\rho)=0$ and thus furnish the numerical values of $\rho_{1}, \rho_{2}, A$, and $B$ necessary to factor $F(\rho)$ into the form

$$
F(\rho) \equiv-2 \alpha_{1}\left(\rho-\rho_{1}\right)\left(\rho_{2}-\rho\right)\left(\rho^{2}+A \rho+B\right),
$$

where $\rho_{1}$ and $\rho_{2}$ are the zeros of $F(\rho)$ closest to the values $r_{1}$ and $r_{2}$. Then, in the intermediary orbit, $\rho$ is restricted to the interval $\rho_{1} \leqq \rho \leqq \rho_{2}$ between two spheroids.

By equating the coefficients of corresponding powers of $\rho$ in (3.11) and (2.8) we find

$$
\begin{array}{ll}
\rho^{3}: & \rho_{1}+\rho_{2}-A=-\mu \alpha_{1}^{-1}=2 a_{0}, \\
\rho^{2}: & B+\rho_{1} \rho_{2}-\left(\rho_{1}+\rho_{2}\right) A=c^{2}-\frac{1}{2} \alpha_{2}^{2} \alpha_{1}^{-1}=c^{2}+a_{0} p_{0}, \\
\rho: & \left(\rho_{1}+\rho_{2}\right) B-\rho_{1} \rho_{2} A=-\mu c^{2} \alpha_{1}^{-1}=2 a_{0} c^{2}, \\
\rho^{0}: & \rho_{1} \rho_{2} B=-\frac{1}{2} c^{2}\left(\alpha_{2}^{2}-\alpha_{3}^{2}\right) \alpha_{1}^{-1}=a_{0} p_{0} c^{2} \sin ^{2} i_{0},
\end{array}
$$

with use of (3.3) through (3.7).

By beginning with the zero-order solution $A=B=0, \quad \rho_{1}+\rho_{2}=r_{1}+r_{2}=2 a_{0}, \quad \rho_{1} \rho_{2}=r_{1} r_{2}=a_{0} p_{0}$, one can solve this set of equations for the four unknowns $A, B, \rho_{1}+\rho_{2}$, and $\rho_{1} \rho_{2}$, by a method of successive approximations. If

$$
\begin{aligned}
& k_{0} \equiv c^{2} / p_{0}^{2} \equiv\left(r_{e} / p_{0}\right)^{2} J_{2}, \\
& x \equiv\left(1-e_{0}^{2}\right)^{\frac{1}{2}}, \\
& y \equiv \alpha_{3} / \alpha_{2}=\cos i_{0},
\end{aligned}
$$


the second-order solution, through $k_{0}^{2}$, is

$$
\begin{aligned}
A & =-2 k_{0} p_{0} y^{2}\left[1+k_{0}\left(2 x^{2}-3 x^{2} y^{2}-4+8 y^{2}\right)+\ldots .\right], \\
B & =k_{0} p_{0}^{2}\left(1-y^{2}\right)\left[1+k_{0}\left(4 y^{2}-x^{2} y^{2}\right)+\ldots .\right], \\
\rho_{1}+\rho_{2} & =2 p_{0} x^{-2}\left[1-k_{0} x^{2} y^{2}-k_{0}^{2} x^{2} y^{2}\left(2 x^{2}-3 x^{2} y^{2}-4+8 y^{2}\right)+\ldots .\right], \\
\rho_{1} \rho_{2} & =p_{0}^{2} x^{-2}\left[1+k_{0} y^{2}\left(x^{2}-4\right)-k_{0}^{2} y^{2}\left(12 x^{2}-x^{4}-20 x^{2} y^{2}-16+32 y^{2}+x^{4} y^{2}\right)+\ldots\right] .
\end{aligned}
$$

The constants

$$
\begin{aligned}
& a \equiv \frac{1}{2}\left(\rho_{1}+\rho_{2}\right), \\
& e=\frac{\rho_{2}-\rho_{1}}{\rho_{2}+\rho_{1}}, \\
& p \equiv a\left(1-e^{2}\right)
\end{aligned}
$$

will occur throughout the evaluation of the $\rho$-integrals. In terms of $a_{0}, e_{0}$, and $i_{0}$, their values, to the second order in $k_{0}$, then satisfy

$$
\begin{aligned}
p / p_{0} & =1+2 k_{0} y^{2}\left(x^{2}-2\right)+k_{0}^{2} y^{2}\left(3 x^{4}-2 x^{4} y^{2}-16 x^{2}+24 x^{2} y^{2}+16-32 y^{2}\right)+\ldots \\
p_{0} / p & =1+2 k_{0} y^{2}\left(2-x^{2}\right)+k_{0}^{2} y^{2}\left(-3 x^{4}+6 x^{4} y^{2}+16 x^{2}-40 x^{2} y^{2}-16+48 y^{2}\right)+\ldots \\
\frac{1-e^{2}}{1-e_{0}^{2}} & =1+k_{0} y^{2}\left(3 x^{2}-4\right)+k_{0}^{2} y^{2}\left(5 x^{4}-2 x^{4} y^{2}-20 x^{2}+28 x^{2} y^{2}+16-32 y^{2}\right)+\ldots \\
\left(\frac{1-e^{2}}{1-e_{0}^{2}}\right)^{\frac{1}{2}} & =1+\frac{1}{2} k_{0} y^{2}\left(3 x^{2}-4\right)+\frac{1}{8} k_{0}^{2} y^{2}\left(20 x^{4}-17 x^{4} y^{2}-80 x^{2}+136 x^{2} y^{2}+64-144 y^{2}\right)+\ldots \\
\left(1-e^{2}\right)^{\frac{1}{2}} p^{-1} & = \\
& a_{0}^{-1}\left(1-e_{0}^{2}\right)^{-\frac{1}{2}}\left[1+\frac{1}{2} k_{0} y^{2}\left(4-x^{2}\right)+\frac{1}{8} k_{0}^{2} y^{2}\left(-4 x^{4}+7 x^{4} y^{2}+48 x^{2}\right.\right. \\
& \left.-104 x^{2} y^{2}-64+176 y^{2}\right)+\ldots .
\end{aligned}
$$

In those places where $e$ occurs alone in the theory, i.e., not in the combination $1-e^{2}$, it may of course be found by use of

$$
e \equiv\left[1-\left(1-e^{2}\right)\right]^{\frac{1}{2}}
$$

By (3.28) this results in

$$
e=\left[e_{0}^{2}+k_{0} x^{2} y^{2}\left(4-3 x^{2}\right)+\ldots\right]^{\frac{1}{2}},
$$

so that when $e_{0}$ is comparable to $k_{0}$ it is not feasible to expand $e$ in a power series in $k_{0}$; indeed if $e_{0}=0$, we should need a power series in $k_{0}^{\frac{1}{2}}$.

Direct use of this second-order solution in factoring $F(\rho)$ will lead to $\rho$-integrals that have secular terms correct only to $O\left(k_{0}^{2}\right)$. Since we are aiming at arbitrarily high accuracy for the secular terms, we include it here for other purposes. The most important of these purposes is to furnish information about the orders of various quantities in $k_{0}$; e.g., $A$ and $B$ are both of order $k_{0}$. Such information will be necessary in carrying out the solution of (2.5) through (2.7) for the periodic terms. The second purpose is to furnish a convenient starting point for any investigators who may choose to use $a_{0}, e_{0}$, and $i_{0}$, along with the $\beta$ 's, as orbital elements, and who will therefore have to solve the equation $F(\rho)=0$ numerically. A third purpose is for use in calculating the mean motions to first order, for comparison with other theories.

The quartic $G(\eta)$, which is quadratic in $\eta^{2}$, may be factored either as

$$
G(\eta) \equiv-2 \alpha_{1} c^{2}\left(\eta_{0}^{2}-\eta^{2}\right)\left(\eta_{2}^{2}-\eta^{2}\right)
$$


or as

$$
G(\eta) \equiv\left(\alpha_{2}^{2}-\alpha_{3}^{2}\right) \eta^{4}\left(\eta^{-2}-\eta_{0}^{-2}\right)\left(\eta^{-2}-\eta_{2}^{-2}\right) .
$$

If we use the latter form, we find on comparing (3.34) with (2.9) that $\eta_{0}^{-2}$ and $\eta_{2}^{-2}$ are the roots of the following quadratic equation in $\eta^{-2}$ :

Thus

$$
\left(\alpha_{2}^{2}-\alpha_{3}^{2}\right) \eta^{-4}+\left(2 \alpha_{1} c^{2}-\alpha_{2}^{2}\right) \eta^{-2}-2 \alpha_{1} c^{2}=0 .
$$

where

$$
\begin{gathered}
\eta_{0}^{-2}=\frac{1}{2}\left(\alpha_{2}^{2}-2 \alpha_{1} c^{2}\right)\left(\alpha_{2}^{2}-\alpha_{3}^{2}\right)^{-1}\left(1+W^{\frac{1}{2}}\right), \\
\eta_{2}^{-2}=\frac{1}{2}\left(\alpha_{2}^{2}-2 \alpha_{1} c^{2}\right)\left(\alpha_{2}^{2}-\alpha_{3}^{2}\right)^{-1}\left(1-W^{\frac{1}{2}}\right),
\end{gathered}
$$

From (3.36) and (3.38) it follows that for $\alpha_{1}<0$

$$
\eta_{0}^{2} \leqq \alpha_{2}^{-2}\left(\alpha_{2}^{2}-\alpha_{3}^{2}\right) \leqq 1
$$

and we shall see below that for satellite motion $\eta_{2}^{2} \gg 1$. Since $\eta^{2}$ cannot exceed 1 , it follows that in the actual motion $\eta$ always lies in the interval $-\eta_{0} \leqq \eta \leqq \eta_{0}$ between two hyperboloids.

We readily find that

$$
\begin{aligned}
\eta_{0} & =\left(\sin i_{0}\right)\left[1-\frac{1}{2} k_{0} x^{2} y^{2}+\frac{1}{8} k_{0}^{2} x^{4} y^{2}\left(7 y^{2}-4\right)+\ldots\right], \\
\left(1-\eta_{0}^{2}\right)^{-\frac{1}{2}} & =\left|\sec i_{0}\right|\left[1-\frac{1}{2} k_{0} x^{2}\left(1-y^{2}\right)+\frac{1}{8} k_{0}^{2} x^{4}\left(1-y^{2}\right)\left(5 y^{2}-1\right)+\ldots\right], \\
\eta_{2}^{-2} & =k_{0} x^{2}\left(1-k_{0} x^{2} y^{2}+\ldots\right), \\
\left(\eta_{0} / \eta_{2}\right)^{2} & =k_{0} x^{2}\left(\sin ^{2} i_{0}\right)\left(1-2 k_{0} x^{2} y^{2}+\ldots\right) .
\end{aligned}
$$

Note that $\eta_{2}^{-2} \leqq k_{0} \approx 10^{-3}$, so that $\eta_{2}^{2} \geqq 1000$.

\section{Factoring the Quartics: Orbital Elements a, e, I, $\beta_{1}, \beta_{2}, \beta_{3}$}

If we equate the coefficients of powers of $\eta^{2}$ in (3.33) with those of corresponding powers in $(2.9)$, we find

$$
\begin{aligned}
\eta_{0}^{2}+\eta_{2}^{2} & =1-\frac{\alpha_{2}^{2}}{2 \alpha_{1} c^{2}}=1+a_{0} p_{0} / c^{2}, \\
\eta_{0}^{2} \eta_{2}^{2} & =-\frac{\alpha_{2}^{2}-\alpha_{3}^{2}}{2 \alpha_{1} c^{2}}=\frac{a_{0} p_{0}}{c^{2}} \sin ^{2} i_{0} .
\end{aligned}
$$

If in (3.12) through (3.15) we use (3.23) through (3.25), we find

$$
\begin{gathered}
2 a-A=2 a_{0}, \\
B+a p-2 A a=c^{2}+a_{0} p_{0}, \\
2 a B-A a p=2 a_{0} c^{2}, \\
B a p=a_{0} p_{0} c^{2} \sin ^{2} i_{0} .
\end{gathered}
$$

Suppose we now regard $a, e$, and

$$
\eta_{0} \equiv \sin I
$$

as known. (When we adopt $a, e, I$, and the $\beta$ 's as orbital elements we are certainly assuming so; we discuss later how they may be determined.) Then in treating the $\rho$-integrals we have five unknowns, viz, $a_{0}, e_{0}, i_{0}, A$, and $B$, and in treating the $\eta$-integrals one additional unknown, viz, $\eta_{2}$. Altogether then, we have six unknowns and we have six equations with which to determine them, (4.1) through (4.6). With these orbital elements, viz, $a, e$, and $\eta_{0}$, however, it 
turns out that the system can be solved exactly and with considerable ease, for the required unknowns. This property of $a, e$, and $\eta_{0}$ was first pointed out by Izsak [4].

To carry out the solution, first eliminate $\eta_{2}$ from (4.1) and (4.2). The result is

$$
\frac{\alpha_{2}^{2}-\alpha_{3}^{2}}{\alpha_{2}^{2}} \equiv \sin ^{2} i_{0}=\eta_{0}^{2}+\frac{c^{2} \eta_{0}^{2}\left(1-\eta_{0}^{2}\right)}{a_{0} p_{0}} .
$$

On inserting (4.8) into (4.6), we find

$$
B a p=c^{2} \eta_{0}^{2}\left[a_{0} p_{0}+c^{2}\left(1-\eta_{0}^{2}\right)\right] .
$$

If we now use (4.3) to eliminate $a_{0}$ from (4.5) and (4.9) to eliminate $a_{0} p_{0}$ from (4.4), we find a pair of simultaneous linear equations for $A$ and $B$ :

Their solution is

$$
\begin{gathered}
\left(a p-c^{2}\right) A-2 a B=-2 a c^{2}, \\
2 c^{2} \eta_{0}^{2} a A+\left(a p-c^{2} \eta_{0}^{2}\right) B=c^{2} \eta_{0}^{2}\left(a p-c^{2} \eta_{0}^{2}\right) .
\end{gathered}
$$

Then, from (4.3) and (4.12)

$$
\begin{aligned}
& A=-\frac{2 a c^{2}\left(1-\eta_{0}^{2}\right)\left(a p-c^{2} \eta_{0}^{2}\right)}{\left(a p-c^{2}\right)\left(a p-c^{2} \eta_{0}^{2}\right)+4 a^{2} c^{2} \eta_{0}^{2}} \\
& B=c^{2} \eta_{0}^{2} \frac{\left(a p-c^{2}\right)\left(a p-c^{2} \eta_{0}^{2}\right)+4 a^{2} c^{2}}{\left(a p-c^{2}\right)\left(a p-c^{2} \eta_{0}^{2}\right)+4 a^{2} c^{2} \eta_{0}^{2}} .
\end{aligned}
$$

$$
-\frac{\mu}{2 a \alpha_{1}} \equiv \frac{a_{0}}{a}=1-\frac{A}{2 a}=1+\frac{c^{2}\left(1-\eta_{0}^{2}\right)\left(a p-c^{2} \eta_{0}^{2}\right)}{\left(a p-c^{2}\right)\left(a p-c^{2} \eta_{0}^{2}\right)+4 a^{2} c^{2} \eta_{0}^{2}}
$$

and from (4.9) and (4.13)

$$
-\frac{\alpha_{2}^{2}}{2 \alpha_{1}} \equiv a_{0} p_{0}=-c^{2}\left(1-\eta_{0}^{2}\right)+a p \frac{\left(a p-c^{2}\right)\left(a p-c^{2} \eta_{0}^{2}\right)+4 a^{2} c^{2}}{\left(a p-c^{2}\right)\left(a p-c^{2} \eta_{0}^{2}\right)+4 a^{2} c^{2} \eta_{0}^{2}}
$$

Equation (4.8) and the relation $\eta_{0} \equiv \sin I$ give

$$
\alpha_{3}=\alpha_{2}\left(1-\frac{c^{2} \eta_{0}^{2}}{a_{0} p_{0}}\right)^{\frac{1}{2}} \cos I
$$

so that (4.15) and (4.15a) determine $\alpha_{3}$. Finally, to obtain $\eta_{2}$, combine (4.2) and (4.6) to obtain $\eta_{2}^{-2}=c^{4} \eta_{0}^{2}(B a p)^{-1}$ and then use (4.13). The result is

$$
\eta_{2}^{-2}=\frac{c^{2}}{a p} \frac{\left(a p-c^{2}\right)\left(a p-c^{2} \eta_{0}^{2}\right)+4 a^{2} c^{2} \eta_{0}^{2}}{\left(a p-c^{2}\right)\left(a p-c^{2} \eta_{0}^{2}\right)+4 a^{2} c^{2}} .
$$

This completes the solution for the required unknowns when the orbital elements are $a, e$, and $I$. In terms of these orbital elements we can now factor the two quartics $F(\rho)$ and $G(\eta)$ exactly and thus evaluate all the integrals.

With use of the old oblateness parameter $k_{0} \equiv c^{2} / p_{0}^{2}$ and a new one, suitable for use with this second set of orbital elements, viz,

$$
k \equiv c^{2} / p^{2} \equiv\left(r_{e} / p\right)^{2} J_{2},
$$

we can readily show that, to the first order, the equations of sections 3 and 4 give similar results. Thus we readily obtain

$$
\begin{aligned}
& A \approx-2 k_{0} p_{0} \cos ^{2} i_{0} \approx-2 k p \cos ^{2} I, \\
& B \approx k_{0} p_{0}^{2} \sin ^{2} i_{0} \approx k p^{2} \sin ^{2} I, \\
& \frac{a_{0}}{a} \approx 1+k_{0}\left(1-e_{0}^{2}\right) \cos ^{2} i_{J} \approx 1+k\left(1-e^{2}\right) \cos ^{2} I,
\end{aligned}
$$




$$
\begin{aligned}
& \frac{p_{0}}{p} \approx 1+2 k_{0}\left(1+e_{0}^{2}\right) \cos ^{2} i_{0} \approx 1+2 k\left(1+e^{2}\right) \cos ^{2} I, \\
& \frac{1-e_{0}^{2}}{1-e^{2}} \approx 1+k_{0}\left(1+3 e_{0}^{2}\right) \cos ^{2} i_{0} \approx 1+k\left(1+3 e^{2}\right) \cos ^{2} I, \\
& \frac{\sin i_{0}}{\sin I} \approx 1+\frac{1}{2} k_{0}\left(1-e_{0}^{2}\right) \cos ^{2} i_{0} \approx 1+\frac{1}{2} k\left(1-e^{2}\right) \cos ^{2} I, \\
& \eta_{2}^{-2} \approx k_{0}\left(1-e_{0}^{2}\right)+\ldots \approx k\left(1-e^{2}\right)+\ldots . \\
& \left(\eta_{0} / \eta_{2}\right)^{2} \approx k_{0}\left(1-e_{0}^{2}\right) \sin ^{2} i_{0} \approx k\left(1-e^{2}\right) \sin ^{2} I+\ldots .
\end{aligned}
$$

With either set of orbital elements, the final solution will be given in terms of $a, e, I, \beta_{1}$, $\beta_{2}$, and $\beta_{3}$, and certain angles $E, v$, and $\psi$, analogous respectively to the eccentric anomaly, the true anomaly, and the argument of latitude in elliptic motion. Once one knows $a$, $e$, and $I$, one can then determine the $\beta$ 's by observing, at various times, whatever quantities will best serve to determine $E, v$, and $\psi$.

If one is using $a_{0}, e_{0}$, and $i_{0}$ as elements, one has to determine them from initial values or from some procedure equivalent to determining initial values. One then has to factor the quartic $F(\rho)$ numerically to determine $a$, $e$, and $\eta_{0} \equiv \sin I$.

If one is using $a, e$, and $I$ as elements, one has to determine them by following the orbit for many revolutions and then applying some sort of iterated least-square process. In this case one can then find the Jacobi constants $\alpha_{1}, \alpha_{2}$, and $\alpha_{3}$ and thus $a_{0}, e_{0}$, and $i_{0}$ by means of the equations of this section.

In any event, the determination of orbital elements, by comparison of theory and observation, is ordinarily considered a completely separate problem in celestial mechanics from the calculation of the motion for given orbital elements. We have included the above remarks only to aid in the possible application of the present theoretical solution. Indeed the problem is further complicated by the small perturbations that occur in practice.

In the rest of the paper we simply assume $a, e, \eta_{0} \equiv \sin I, \beta_{1}, \beta_{2}$, and $\beta_{3}$ to be known and then complete the solution for the intermediary orbit. There will be one restriction, however. The method of evaluating the $\rho$-integrals will be found to depend on the orbital inclination $i_{0}$ or $I$. For values of $I$ less than $1^{\circ} 54^{\prime}$ or greater than $178^{\circ} 6^{\prime}$, a different approach would be needed. We shall therefore restrict considerations in the present paper to orbits that have inclinations between these two values, thereby ruling out equatorial or almost equatorial orbits.

\section{The $\rho$-Integrals}

In (2.5) through (2.7) the $\rho$-integrals are

$$
\begin{aligned}
& R_{1} \equiv \pm \int_{\rho_{1}}^{\rho} \rho^{2} F^{-\frac{1}{2}}(\rho) d \rho \\
& R_{2} \equiv \pm \int_{\rho_{1}}^{\rho} F^{-\frac{1}{2}}(\rho) d \rho \\
& R_{3} \equiv \pm \int_{\rho_{1}}^{\rho}\left(\rho^{2}+c^{2}\right)^{-1} F^{-\frac{1}{2}}(\rho) d \rho
\end{aligned}
$$

where $F(\rho)$ is given by (3.11), $A$ and $B$ by (4.12) and (4.13), $p$ by (3.25), and $\rho_{1}$ and $\rho_{2}$ by

$$
\rho_{1}=a(1-e), \quad \rho_{2}=a(1+e) .
$$

Equations (5.4) follow from (3.23) and (3.24). Then

$$
F^{-\frac{1}{2}}(\rho)=\left(-2 \alpha_{1}\right)^{-\frac{1}{2}}\left[\left(\rho-\rho_{1}\right)\left(\rho_{2}-\rho\right)\right]^{-\frac{1}{2}} \rho^{-1}\left(1+A \rho^{-1}+B \rho^{-2}\right)^{-\frac{1}{2}} .
$$


If, for convenience, we put

and

$$
A \equiv-2 b_{1}, \quad B \equiv b_{2}^{2}, \quad \lambda \equiv b_{1} / b_{2}
$$

then

$$
h \equiv b_{2} / \rho,
$$

if $|h|<1$ and $|\lambda|<1$.

$$
\begin{aligned}
\left(1+A \rho^{-1}+B \rho^{-2}\right)^{-\frac{1}{2}} & \equiv\left(1-2 \lambda h+h^{2}\right)^{-\frac{1}{3}} \\
& =\sum_{n=0}^{\infty} h^{n} P_{n}(\lambda),
\end{aligned}
$$

The expansion (5.9) will be a convenient tool for evaluating all the $\rho$-integrals, whenever it can be used. To see when, use (5.6), (5.7), (4.18), and (4.19). Then to $O(k)$

so that $b_{2} / \rho \ll 1$ and

$$
b_{1}=k p \cos ^{2} I, \quad b_{2}=k^{\frac{1}{2}} p \sin I,
$$

Thus to $O(k),|\lambda|<1$ whenever

$$
\lambda=k^{\frac{1}{2}} \cos ^{2} I \csc I>0 .
$$

or whenever

$$
k^{\frac{1}{2}} \cos ^{2} I \csc I<1,
$$

$$
\tan ^{2} I+\tan ^{4} I>k
$$

or

$$
2 \tan ^{2} I>(1+4 k)^{\frac{1}{2}}-1 .
$$

To $O(k)$, this becomes

$$
\tan ^{2} I>k \text {. }
$$

But $k=\left(r_{e} / p\right)^{2} J_{2}$ and $J_{2}=(1.08) 10^{-3}$ for the earth, so that $\lambda<1$ whenever

$$
|\tan I|>0.033 r_{e} / p
$$

For close orbits $r_{e} / p \approx 1$, so that the necessary condition becomes

$$
I_{c}<I<180^{\circ}-I_{c}, \quad I_{c} \approx 1^{\circ} 54^{\prime} .
$$

The expansion will thus work for all satellite orbits that are inclined more than $1^{\circ} 54^{\prime}$ to the equator. For those orbits that lie closer to the equator one must use some other method to evaluate the $\rho$-integrals. On inserting (5.7) and (5.9) into (5.5), we find

$$
F(\rho)^{-\frac{1}{2}}=\left(-2 \alpha_{1}\right)^{-\frac{1}{2}} \sum_{n=0}^{\infty} b_{2}^{n} P_{n}(\lambda) \rho^{-1-n}\left[\left(\rho-\rho_{1}\right)\left(\rho_{2}-\rho\right)\right]^{-\frac{1}{2}}
$$

With use of (5.1), (5.10), and (5.6), we find

$$
\left(-2 \alpha_{1}\right)^{\frac{1}{2}} R_{1}=\int_{\rho_{1}}^{\rho}\left(\rho+b_{1}\right)\left[\left(\rho-\rho_{1}\right)\left(\rho_{2}-\rho\right)\right]^{-\frac{1}{2}}( \pm d \rho)+\sum_{n=2}^{\infty} b_{2}^{n} P_{n}(\lambda) \int_{\rho_{1}}^{\rho} \rho^{1-n}\left[\left(\rho-\rho_{1}\right)\left(\rho_{2}-\rho\right)\right]^{-\frac{1}{2}}( \pm d \rho) .
$$

Each of the separate integrals in (5.11) is a multiple-valued function of $\rho$. It is appropriate to change variables in each to a uniformizing variable $E$ or $v$, analogous respectively to the eccentric and true anomalies in elliptic motion. We define $E$ and $v$ by requiring them to satisfy

$$
\rho=a(1-e \cos E)=(1+e \cos v)^{-1} p
$$

and always to increase with time. Then, from (5.12), (5.4), and (3.23) through (3.25), we find

$$
\left[\left(\rho-\rho_{1}\right)\left(\rho_{2}-\rho\right)\right]^{-\frac{1}{2}}( \pm d \rho)=d E=\left(1-e^{2}\right)^{\frac{1}{2}}(1+e \cos v)^{-1} d v
$$




\subsection{The Integral $R_{1}$}

On introducing $E$ into the first integral in (5.11) and $v$ into each of the integrals in the series, we find

$$
\left(-2 \alpha_{1}\right)^{\frac{1}{2}} R_{1}=b_{1} E+a(E-e \sin E)+\left(1-e^{2}\right)^{\frac{1}{2}} p \sum_{n=2}^{\infty}\left(b_{2} / p\right)^{n} P_{n}(\lambda) \int_{0}^{0}(1+e \cos v)^{n-2} d v .
$$

To investigate the convergence of the series we write

$$
\begin{aligned}
S_{1} & \equiv \sum_{n=2}^{\infty}\left(b_{2} / p\right)^{n} P_{n}(\lambda) \int_{0}^{v}(1+e \cos v)^{n-2} d v \\
& =\left(b_{2} / p\right)^{2} \sum_{m=0}^{\infty}\left(b_{2} / p\right)^{m} P_{m+2}(\lambda) \int_{0}^{v}(1+e \cos v)^{m} d v .
\end{aligned}
$$

Then

$$
\left|S_{1}\right| \leqq\left(b_{2} / p\right)^{2} \sum_{m=0}^{\infty}\left(b_{2} / p\right)^{m}\left|P_{m+2}(\lambda)\right|(1+e)^{m} v
$$

and since $\left|P_{n}(\lambda)\right| \leqq 1$ for all $|\lambda| \leqq 1$, we have

$$
\begin{aligned}
\left|S_{1}\right| & \leqq\left(b_{2} / p\right)^{2} v \sum_{m=0}^{\infty}\left[\frac{b_{2}(1+e)}{p}\right]^{m} \\
& \leqq \frac{\left(b_{2} / p\right)^{2} v}{1-b_{2}(1+e) / p} .
\end{aligned}
$$

By (4.19) and (5.6), however, $b_{2} / p \approx k^{\frac{1}{2}} \sin I$ and of course $1+e \leqq 2$, so that

$$
b_{2}(1+e) / p \leqq 2 k^{\frac{1}{2}} \sin I \leqq 2 k^{\frac{1}{2}} .
$$

Since $k \approx 10^{-3}$, we have $b_{2}(1+e) / p \leqq 0.063$. Thus the series $S_{1}$ converges, and converges more rapidly than a geometric series of common ratio $\approx 1 / 16$. Actually, since we have shown that the series $S_{1}$ converges absolutely, we can regroup it into the sum of a series $S_{1 e}$ containing only the even values of $n$ and a series $S_{10}$ containing only the odd values of $n$. It is then a simple matter to show that $S_{1} \equiv S_{1 e}+S_{10}$ converges more rapidly than the geometric series $\left[1+b_{2}(1+e) / p\right]$. $\sum_{n=0}^{\infty}\left[b_{2}(1+e) / p\right]^{2 n}$. That is, we can actually expect the convergence of $S_{1}$ to be as rapid as that of a geometric series of common ratio $\left[b_{2}(1+e) / p\right] \leqq 4 k \approx 1 / 250$.

To decompose the series $S_{1}$ into a part proportional to $v$ and a periodic part, note first that if

$$
f_{m}(v) \equiv \int_{0}^{v}(1+e \cos v)^{m} d v
$$

then $f_{n}(v)-(2 \pi)^{-1} f_{m}(2 \pi) v$ is an odd function of $v$, of period $2 \pi$. But $f_{m}(2 \pi)=2 f_{m}(\pi)$, so that we obtain

$$
f_{m}(v) \equiv \int_{0}^{v}(1+e \cos v)^{m} d v=\pi^{-1} v \int_{0}^{\pi}(1+e \cos v)^{m} d v+\sum_{j=1}^{\infty} c_{m j} \sin j v
$$

To obtain, to any order in $k$, the parts of the $\rho$-integrals proportional to $v$, we shall need to consider all integral values of $m$ in $\pi^{-1} f_{m}(\pi)$. To obtain the periodic parts correct to order $k^{2}$, we shall need values of $m$ only up to 4 .

To obtain a convenient expression for the $v$-term of $f_{m}(v)$, note that [5]

$$
\int_{0}^{\pi}\left(z+\sqrt{z^{2}-1} \cos v\right)^{m} d v=\pi P_{m}(z)
$$

for all values of $z$, including real values greater than unity. Here $P_{m}(z)$ is the Legendre polynomial

$$
P_{m}(z) \equiv \frac{2^{-m}}{m !} \frac{d^{m}}{d z^{m}}\left(z^{2}-1\right)^{m}
$$


of the same polynomial form in $z$ that holds when $|z|<1$, when it can be defined by the usual generating function. If we put

in $(5.23)$, we find

$$
z=\left(1-e^{2}\right)^{-\frac{1}{2}}
$$

where

$$
\begin{aligned}
\int_{0}^{\pi}(1+e \cos v)^{m} d v & =\pi\left(1-e^{2}\right)^{m / 2} P_{m}\left[\left(1-e^{2}\right)^{-\frac{1}{2}}\right] \\
& =\pi R_{m}\left(\sqrt{1-e^{2}}\right)
\end{aligned}
$$

$$
R_{m}(x) \equiv x^{m} P_{m}(1 / x) \quad(0 \leqq x \leqq 1) .
$$

\begin{tabular}{|c|c|}
\hline$m$ & $R_{m}(x)$ \\
\hline $\begin{array}{l}0 \\
1 \\
2 \\
3 \\
4 \\
5 \\
5 \\
6\end{array}$ & $\begin{array}{l}1 \\
1 \\
1 / 2\left(3-x^{2}\right) \\
1 / 2\left(5-3 x^{2}\right) \\
(1 / 8)\left(35-30 x^{2}+3 x^{4}\right) \\
(1 / 8)\left(63-70 x^{2}+15 x^{4}\right) \\
(1 / 16)\left(231-315 x^{2}+105 x^{4}-5 x^{5}\right)\end{array}$ \\
\hline
\end{tabular}

Thus $R_{m}(x)$ is a polynomial of degree $[m / 2]$ in $x^{2}$. The first few of these polynomials are given in table 1.

TABLE 1

From (5.22) and (5.27) we then obtain

$$
\int_{0}^{v}(1+e \cos v)^{m} d v=R_{m}\left(\sqrt{1-e^{2}}\right) v+\sum_{j=1}^{\infty} c_{m j} \cdot \sin j v
$$

Through $m=4$, the coefficients $c_{m j}$ are easy to find, simply by binomial expansion of the integrand and conversion to a trigonometric polynomial.

The results are given in the following table:

TaBle 2. Coefficients $c_{m i}$ in (5.29)

\begin{tabular}{c|c|c|c|c}
\hline \hline$j$ & 1 & 2 & 3 & 4 \\
& & & & \\
\hline 0 & & & & \\
1 & 1 & & & \\
2 & $2 e$ & $e^{2 / 4}$ & & \\
3 & $3 e+3 e^{3} / 4$ & $3 e^{2 / 4}$ & $e^{3 / 12}$ & \\
4 & $4 e+3 e^{3}$ & $3 e^{2} / 2+e^{4} / 4$ & $e^{3} / 3$ & $e^{4} / 32$ \\
\hline
\end{tabular}

In (5.14), $b_{2}^{n}=O\left(k^{n / 2}\right)$ and $P_{n}(\lambda)=O\left(\lambda^{0}\right)=O\left(k^{0}\right)$ if $n$ is even or $P_{n}(\lambda)=O(\lambda)=O\left(k^{\frac{1}{2}}\right)$ if $n$ is odd. On inserting (5.29) into (5.14), using table 2, and keeping periodic terms through $O\left(k^{2}\right)$ only, we find

where

$$
\left(-2 \alpha_{1}\right)^{\frac{1}{2}} R_{1}=b_{1} E+a(E-e \sin E)+A_{1} v+\sum_{j=1}^{2} A_{1 \jmath} \cdot \sin j v
$$

and

$$
A_{1}=\left(1-e^{2}\right)^{\frac{1}{2}} p \sum_{n=2}^{\infty}\left(b_{2} / p\right)^{n} P_{n}(\lambda) R_{n-2}\left(\sqrt{1-e^{2}}\right)
$$

$$
\begin{aligned}
& A_{11}=\frac{3\left(1-e^{2}\right)^{\frac{1}{2}}}{4 p^{3}}\left(-2 b_{1} b_{2}^{2} p+b_{2}^{4}\right) e, \\
& A_{12}=\frac{3\left(1-e^{2}\right)^{\frac{1}{2}}}{32 p^{3}} b_{2}^{4} e^{2} .
\end{aligned}
$$

The above proof of convergence of the series for $R_{1}$ also shows the rapid convergence of the series (5.31) for the coefficient $A_{1}$ of the $v$-term. 


\subsection{The Integral $R_{2}$}

On inserting (5.10) into (5.2) and using (5.12) and (5.13), we find

$$
\left(-2 \alpha_{1}\right)^{\frac{1}{2}} R_{2}=\left(1-e^{2}\right)^{\frac{1}{2}} p^{-1} \sum_{n=0}^{\infty}\left(b_{2} / p\right)^{n} P_{n}(\lambda) \int_{0}^{v}(1+e \cos v)^{n} d v .
$$

As above, one can show at once that this series converges more rapidly than the geometric series $v \sum_{n=0}^{\infty}\left[b_{2}(1+e) / p\right]^{n}$, with common ratio $b_{2}(1+e) / p \leqq 2 k^{\frac{1}{2}} \approx 0.066$. The same proof then applies to the coefficient $A_{2}$ in

$$
\left(-2 \alpha_{1}\right)^{\frac{1}{2}} R_{2}=A_{2} v+\sum_{j=1}^{4} A_{2 j} \sin j v .
$$

Using the same methods as for $R_{1}$, we then find

$$
A_{2}=\left(1-e^{2}\right)^{\frac{1}{2}} p^{-1} \sum_{n=0}^{\infty}\left(b_{2} / p\right)^{n} P_{n}(\lambda) R_{n}\left(\sqrt{1-e^{2}}\right)
$$

and, through periodic terms of $O\left(k^{2}\right)$ :

$$
\begin{aligned}
& A_{21}=\left(1-e^{2}\right)^{\frac{1}{2}} p^{-1} e\left[b_{1} p^{-1}+\left(3 b_{1}^{2}-b_{2}^{2}\right) p^{-2}-\frac{9}{2} b_{1} b_{2}^{2}\left(1+e^{2} / 4\right) p^{-3}+\frac{3}{8} b_{2}^{4}\left(4+3 e^{2}\right) p^{-4}\right], \\
& A_{22}=\left(1-e^{2}\right)^{\frac{1}{2}} p^{-1}\left[\frac{e^{2}}{8}\left(3 b_{1}^{2}-b_{2}^{2}\right) p^{-2}-\frac{9}{8} e^{2} b_{1} b_{2}^{2} p^{-3}+\frac{3 b_{2}^{4}}{32}\left(6 e^{2}+e^{4}\right) p^{-4}\right], \\
& A_{23}=\left(1-e^{2}\right)^{\frac{1}{2}} p^{-1} \frac{e^{3}}{8}\left(-b_{1} b_{2}^{2} p^{-3}+b_{2}^{4} p^{-4}\right), \\
& A_{24}=\left(1-e^{2}\right)^{\frac{1}{2}} p^{-5} \frac{3}{256} b_{2}^{4} e^{4} .
\end{aligned}
$$

\subsection{The Integral $R_{3}$}

If in (5.3) we now use the binomial expansion

$$
\left(\rho^{2}+c^{2}\right)^{-1}=\rho^{-2} \sum_{j=0}^{\infty}(-1)^{j} c^{2 j} \rho^{-2 j}
$$

and insert the expressions (5.10), (5.12), and (5.13), we find

$$
\left(-2 \alpha_{1}\right)^{\frac{1}{2}} R_{3}=\left(1-e^{2}\right)^{\frac{1}{2}} p^{-3} \int_{0}^{v} \sum_{j=0}^{\infty}(-1)^{j}(c / p)^{2 j}(1+e \cos v)^{2 j} \sum_{n=0}^{\infty}\left(b_{2} / p\right)^{n} P_{n}(\lambda)(1+e \cos v)^{n+2} d v,
$$

where the integrand is the product of two series, each of which converges absolutely for any value of $v$. Then [6] it is equal to the series formed by summing the products of the individual terms, taken in any order, and this resulting series is itself absolutely convergent, for any value of $v$. It is therefore uniformly convergent, by the Weierstress $M$-test [7], so that it may be integrated term by term.

Let us now rewrite $(5.42)$ in the form

$$
\left(-2 \alpha_{1}\right)^{\frac{1}{2}} R_{3}=\left(1-e^{2}\right)^{\frac{1}{2}} p^{-3} \int_{0}^{v} \sum_{m=0}^{\infty} D_{m}(1+e \cos v)^{m+2} d v .
$$

Here

$$
D_{m}=\sum d_{j} \delta_{n^{\prime}}
$$

summed over all those non-negative integral values of $j$ and $n^{\prime}$ for which

$$
2 j+n^{\prime}=m,
$$


and where

$$
\begin{aligned}
d_{j} & =(-1)^{j}(c / p)^{2 j}, \\
\delta_{n^{\prime}} & =\left(b_{2} / p\right)^{n^{\prime}} P_{n^{\prime}}(\lambda) .
\end{aligned}
$$

Then, because of the uniform convergence,

$$
\left(-2 \alpha_{1}\right)^{\frac{1}{2}} R_{3}=\left(1-e^{2}\right)^{\frac{1}{2}} p^{-3} \sum_{m=0}^{\infty} D_{m} \int_{0}^{v}(1+e \cos v)^{m+2} d v .
$$

It is interesting to investigate here the rapidity of convergence of the series

$$
S \equiv \sum_{n=0}^{\infty} D_{m} \int_{0}^{v}(1+e \cos v)^{m+2} d v .
$$

If $m$ is even, we have $m=2 i$ and $n^{\prime}=2 n$, so that $2 j+2 n=2 i$ and $j=i-n$. Then

$$
D_{m}=D_{2 i}=\sum_{n=0}^{i} d_{i-n} \delta_{2 n}=\sum_{n=0}^{i}(-1)^{i-n}(c / p)^{2 i-2 n}\left(b_{2} / p\right)^{2 n} P_{2 n}(\lambda)
$$

so that

$$
\left|D_{2 i}\right| \leqq(c / p)^{2 i} \sum_{n=0}^{i}\left(b_{2} / c\right)^{2 n}
$$

But $c^{2}=k p^{2}$ and $b_{2}^{2} \approx k p^{2} \sin ^{2} I$, by (4.19), so that

Then

$$
\left(b_{2} / c\right)^{2} \approx \sin ^{2} I \leqq 1 .
$$

$$
\left|D_{2 i}\right| \leqq(i+1) k^{i} .
$$

If $m$ is odd, we have $m=2 i+1$ and $n^{\prime}=2 n+1$, so that

Then

$$
D_{2 i+1}=\sum_{n=0}^{i} d_{i-n} \delta_{2 n+1}=\sum_{n=0}^{i}(-1)^{i-n}(c / p)^{2 i-2 n}\left(b_{2} / p\right)^{2 n+1} P_{2 n+1}(\lambda) .
$$

$$
\left|D_{2 i+1}\right| \leqq(c / p)^{2 i}\left(b_{2} / p\right) \sum_{n=0}^{i}\left(b_{2} / c\right)^{2 n} \leqq k^{i} k^{\frac{1}{2}}(i+1) \sin I .
$$

Then, breaking up $S$ into an even series $S_{e}$ and an odd series $S_{o}$, we find

$$
\left|S_{e}\right| \leqq \sum_{i=0}^{\infty}(i+1) k^{i}(1+e)^{2 i+2} v \leqq(1+e)^{2} v \sum_{i=0}^{\infty}(i+1)\left[k(1+e)^{2}\right]^{i} .
$$

Using $\sum_{0}^{\infty} x^{i}=(1-x)^{-1}$ and $\sum_{1}^{\infty} i x^{i}=x(1-x)^{-2}$, we find $\sum_{0}^{\infty}(i+1) x^{i}=(1-x)^{-2}$, so that

Similarly

$$
\left|S_{e}\right| \leqq \frac{(1+e)^{2} v}{\left[1-k\left(1+e^{2}\right)\right]^{2}}
$$

$$
\begin{aligned}
\left|S_{o}\right| & \leqq k^{\frac{1}{2}}(1+e)^{3} v \sin I \sum_{i=0}^{\infty}(i+1)\left[k(1+e)^{2}\right]^{i} \\
& \leqq \frac{k^{\frac{1}{2}}(1+e)^{3} v \sin I}{\left[1-k(1+e)^{2}\right]^{2}}
\end{aligned}
$$

Thus,

$$
|S| \leqq \frac{(1+e)^{2} v\left[1+k^{\frac{1}{2}}(1+e) \sin I\right]}{\left[1-k(1+e)^{2}\right]^{2}} .
$$

The series (5.49) for $R_{3}$ thus converges more rapidly than the series expansion of the function given in (5.59). 
Through $O\left(k^{2}\right)$ the values of $D_{m}$ are given by

TABLE 3

\begin{tabular}{|c|c|c|}
\hline$m$ & Order & $D_{m}$ \\
\hline $\begin{array}{l}0 \\
1 \\
2 \\
3 \\
4\end{array}$ & $\begin{array}{c}k^{0} \\
k \\
k \\
k^{2} \\
k^{2}\end{array}$ & $\begin{aligned} d_{0} \delta_{0} & =1 \\
d_{0} \delta_{1} & =\delta_{1}=b_{1} / p \approx k \cos ^{2} I \\
d_{0} \delta_{2}+d_{1} \delta_{0} & =\left(b_{2} / p\right)^{2} P_{2}\left(b_{1} / b_{2}\right)-(c / p)^{2} \\
d_{0} \delta_{3}+d_{1} \delta_{1} & =\left(b_{2} / p\right)^{2} P_{3}\left(b_{1} / b_{2}\right)-(c / p)^{2}\left(b_{1} / p\right) \\
d_{0} \delta_{4}+d_{1} \delta_{2}+d_{2} \delta_{0} & =\left(b_{2} / p\right)^{4} P_{4}\left(b_{1} / b_{2}\right)-(c / p)^{2}\left(b_{2} / p\right)^{2} P_{2}\left(b_{1} / b_{2}\right)+(c / p)^{4}\end{aligned}$ \\
\hline
\end{tabular}

As with $R_{1}$ and $R_{2}$, we find

$$
\left(-2 \alpha_{1}\right)^{\frac{1}{2}} R_{3}=A_{3} v+\sum_{j=1}^{\infty} A_{3 j} \sin j v
$$

where

$$
A_{3}=\left(1-e^{2}\right)^{\frac{1}{2}} p^{-3} \sum_{m=0}^{\infty} D_{m} R_{m+2}\left(\sqrt{1-e^{2}}\right) .
$$

The remarks about the convergence of the series (5.49) apply also to (5.61).

Since $R_{3}$ is multiplied by $c^{2}=k p^{2}$ in $(2.7)$, we need periodic terms only through $O(k)$, in order to have periodic terms in the final solution correct through $O\left(k^{2}\right)$. By (5.43) and tables 2 and 3 , their coefficients are

$$
\begin{aligned}
& A_{31}=\left(1-e^{2}\right)^{\frac{1}{2}} p^{-3} e\left[2+b_{1} p^{-1}\left(3+\frac{3}{4} e^{2}\right)-p^{-2}\left(\frac{b_{2}^{2}}{2}+c^{2}\right)\left(4+3 e^{2}\right)\right], \\
& A_{32}=\left(1-e^{2}\right)^{\frac{1}{2}} p^{-3}\left[\frac{e^{2}}{4}+\frac{3}{4} \frac{b_{1}}{p} e^{2}-p^{2} \frac{e^{4}}{4}+\frac{3}{2} e^{2}\left(\frac{b_{2}^{2}}{2}+c^{2}\right)\right], \\
& A_{33}=\left(1-e^{2}\right)^{\frac{1}{2}} p^{-3}\left[\frac{e^{3} b^{1}}{12 p}-\frac{e^{3}}{3 p^{2}}\left(\frac{b_{2}^{2}}{2}+c^{2}\right)\right], \\
& A_{34}=\left(1-e^{2}\right)^{\frac{1}{2}} p^{-3}\left[-\frac{e^{4}}{32 p^{2}}\left(\frac{b_{2}^{2}}{2}+c^{2}\right)\right] .
\end{aligned}
$$

\section{The $\eta$-Integrals}

In (2.5) through (2.7) the $\eta$-integrals are

$$
\begin{aligned}
& N_{1}= \pm \int_{0}^{\eta} \eta^{2} G^{-\frac{1}{2}}(\eta) d \eta, \\
& N_{2}= \pm \int_{0}^{\eta} G^{-\frac{1}{2}}(\eta) d \eta, \\
& N_{3}= \pm \int_{0}^{\eta}\left(1-\eta^{2}\right)^{-1} G(\eta)^{-\frac{1}{2}} d \eta,
\end{aligned}
$$

where $G(\eta)$ is given by (3.34), $\eta_{2}$ by (4.16), $\alpha_{2}^{2}$ by (4.14) and (4.15), and $\alpha_{3}^{2}-\alpha_{2}^{2}$ finally by (4.8).

In evaluating $N_{1}$ and $N_{2}$ it is convenient to put

$$
\eta=\eta_{0} \sin \psi,
$$

where $\psi$ is to be an angle that always increases with time. In the limiting case $c=0$ we should have $\sin \psi=\sin \theta / \sin I$ and $\psi$ would thus reduce to the argument of latitude, i.e., to the angle between the line of nodes and the radius vector to the satellite.

Then

$$
\pm G^{-\frac{1}{2}}(\eta) d \eta=\left(\alpha_{2}^{2}-\alpha_{3}^{2}\right)^{-\frac{1}{2}} \eta_{0}\left(1-q^{2} \sin ^{2} \psi\right)^{-\frac{1}{2}} d \psi,
$$


where

$\eta_{2}$ being given by (4.16).

$$
q \equiv \eta_{0} / \eta_{2}=O\left(k^{\frac{1}{2}}\right) \ll 1
$$

\subsection{The Integrals $N_{1}$ and $N_{2}$}

Insertion of (6.4) and (6.5) into (6.1) gives

$$
N_{1}=\left(\alpha_{2}^{2}-\alpha_{3}^{2}\right)^{-\frac{1}{2}} \eta_{0}^{3} \int_{0}^{\psi}\left(1--q^{2} \sin ^{2} \psi\right)^{-\frac{1}{2}} \sin ^{2} \psi d \psi
$$

Because of the identity

$$
\begin{aligned}
& \left(1-q^{2} \sin ^{2} \psi\right)^{-\frac{1}{2}} q^{2} \sin ^{2} \psi \equiv\left(1-q^{2} \sin ^{2} \psi\right)^{-\frac{1}{2}}-\left(1-q^{2} \sin ^{2} \psi\right)^{\frac{1}{2}}, \\
N_{1}= & \left(\alpha_{2}^{2}-\alpha_{3}^{2}\right)^{-\frac{1}{2}} \eta_{0}^{3} q^{-2}\left[\int_{0}^{\psi}\left(1-q^{2} \sin ^{2} \psi\right)^{-\frac{1}{2}} d \psi-\int_{0}^{\psi}\left(1-q^{2} \sin ^{2} \psi\right)^{\frac{1}{2}} d \psi\right] \\
= & \left(\alpha_{2}^{2}-\alpha_{3}^{2}\right)^{-\frac{1}{2}} \eta_{0}^{3} q^{-2}[F(\psi, q)-E(\psi, q)],
\end{aligned}
$$

where $F(\psi, q)$ and $E(\psi, q)$ are respectively the elliptic integrals of the first and second kinds, with modulus $q$.

Insertion of (6.4) and (6.5) into (6.2) gives

$$
\begin{aligned}
N_{2} & =\left(\alpha_{2}^{2}-\alpha_{3}^{2}\right)^{-\frac{1}{2}} \eta_{0} \int_{0}^{\psi}\left(1-q^{2} \sin ^{2} \psi\right)^{-\frac{1}{2}} d \psi \\
& =\left(\alpha_{2}^{2}-\alpha_{3}^{2}\right)^{-\frac{1}{2}} \eta_{0} F(\psi, q) .
\end{aligned}
$$

Thus $N_{1}$ and $N_{2}$ can both be expressed exactly in terms of elliptic integrals of the first and second kinds. Our ultimate purpose, however, is to express each uniformising variable as the sum of an exact secular term and periodic terms correct through $O\left(k^{2}\right)$. For this purpose it is convenient to express each elliptic integral as a linear combination of $\psi$ and a Fourier series $\sum_{1}^{\infty} B_{n} \sin 2 n \psi$

To do so, consider

$$
F(\psi, q) \equiv \int_{0}^{\psi}\left(1-q^{2} \sin ^{2} x\right)^{-\frac{1}{2}} d x
$$

Some simple transformations show that

where

$$
F(\psi+\pi, q)=F(\psi, q)+2 K(q),
$$

$$
K(q) \equiv \int_{0}^{\pi / 2}\left(1-q^{2} \sin ^{2} x\right)^{-\frac{1}{2}} d x
$$

is the complete elliptic integral of the first kind. It follows that the function $F(\psi, q)$ $-(2 / \pi) K(q) \psi$ is periodic in $\psi$ with period $\pi$.

Furthermore it is an odd function of $\psi$, so that it can be expanded in a Fourier series containing only the sines of even multiples of $\psi$. Thus,

$$
F(\psi, q)=(2 / \pi) K(q) \psi+\sum_{m=1}^{\infty} F_{q m} \sin 2 m \psi
$$

To calculate the Fourier coefficients $F_{q m}$, differentiate (6.16) with respect to $\psi$ and use (6.13). Then

$$
\left(1-q^{2} \sin ^{2} \psi\right)^{-\frac{1}{2}}=(2 / \pi) K(q)+2 \sum_{1}^{\infty} m F_{q m} \cos 2 m \psi
$$


The Fourier coefficients $F_{g m}$ are then given by

$$
F_{q m}=(2 / \pi m) \int_{0}^{\pi / 2}\left(1-q^{2} \sin ^{2} x\right)^{-\frac{1}{2}} \cos 2 m x d x .
$$

Since $\cos 2 m x$ is a polynomial in $\cos ^{2} x$, each $F_{q m}$ can ultimately be expressed as a linear combination of $K(q)$ and the complete elliptic integral

$$
E(q) \equiv \int_{0}^{\pi / 2}\left(1-q^{2} \sin ^{2} x\right)^{\frac{1}{2}} d x
$$

Such a procedure, however, would not readily reveal the order of each coefficient in $q^{2}$, which itself is of order $k$. (Actually we shall show that $F_{q m}$ is of order $k^{m}$, so that we shall need only $F_{q 1}$ and $F_{q 2}$.) Instead we expand the radical, obtaining

Then

$$
\left(1-q^{2} \sin ^{2} x\right)^{-\frac{1}{2}}=1+\sum_{n=1}^{\infty} \frac{(2 n) ! q^{2 n} \sin ^{2 n} x}{2^{2 n}(n !)^{2}} .
$$

Also

$$
F_{Q m}=\frac{2}{\pi m} \sum_{n=1}^{\infty} \frac{(2 n) ! q^{2 n}}{2^{n}(n !)^{2}} \int_{0}^{\pi / 2} \sin ^{2 n} x \cos 2 m x d x
$$

$$
\sin ^{2 n} x=\frac{(2 n) !}{(n !)^{2} 2^{n}}+(-1)^{n} 2^{1-2 n} \sum_{j=0}^{n-1}(-1)^{j} \frac{(2 n) !}{(2 n-j) ! j !} \cos (2 n-2 j) x, \quad(n \geqq 1)
$$

and

$$
\cos [(2 n-2 j) x] \cos 2 m x=\frac{1}{2} \cos [(2 n+2 m-2 j) x]+\frac{1}{2} \cos [(2 n-2 m-2 j) x] .
$$

The integral of this product from 0 to $\pi / 2$ fails to vanish only if $j=n+m$ or if $j=n-m$. The value $j=n+m$ is absent from (6.22), so that only the term $j=n-m$ in (6.22) contributes to the integral in (6.21). Thus

$$
\int_{0}^{\pi / 2} \sin ^{2 n} x \cos 2 m x d x=(-1)^{m} 2^{-2 n} \frac{(2 n) !}{(n+m) !(n-m) !} \frac{\pi}{2}, \quad(n \geqq m) .
$$

On inserting (6.24) into (6.21), we find

$$
F_{q m}=(-1)^{m} m^{-1} \sum_{n=m}^{\infty} \frac{(2 n) !^{2} q^{2 n}}{(n+m) !(n-m) ! 2^{4 n}(n !)^{2}} .
$$

Inspection of (6.25) now shows that $F_{2 m}=O\left(q^{2 m}\right)=O\left(k^{m}\right)$. The first two values are, to order $q^{4}$ or order $k^{2}$ :

$$
\begin{aligned}
& F_{q 1}=-\frac{q^{2}}{8}\left(1+\frac{3}{4} q^{2}\right)+\ldots \\
& F_{q 2}=\frac{3 q^{4}}{256}+\ldots
\end{aligned}
$$

For our purposes, therefore,

$$
F(\psi, q)=(2 / \pi) K(q) \psi-\frac{q^{2}}{8}\left(1+\frac{3}{4} q^{2}\right) \sin 2 \psi+\frac{3 q^{4}}{256} \sin 4 \psi+\ldots
$$

Similar considerations about periodicity and oddness show that

$$
E(\psi, q) \equiv \int_{0}^{\psi}\left(1-q^{2} \sin ^{2} x\right)^{\frac{1}{2}} d x=(2 / \pi) E(q) \psi+\sum_{1}^{\infty} E_{q m} \sin 2 m \psi .
$$

Then, as before,

$$
\left(1-q^{2} \sin ^{2} \psi\right)^{\frac{1}{2}}=(2 / \pi) E(q)+2 \sum_{1}^{\infty} m E_{q m} \cos 2 m \psi
$$


and

Also

$$
E_{q m}=(2 / \pi m) \int_{0}^{\pi / 2}\left(1-q^{2} \sin ^{2} x\right)^{\frac{1}{2}} \cos 2 m x d x .
$$

$$
\left(1-q^{2} \sin ^{2} x\right)^{\frac{1}{2}}=1-\sum_{n=1}^{\infty} \frac{(2 n-2) ! q^{2 n} \sin ^{2 n}}{2^{2 n-1} n !(n-1) !} x .
$$

Inserting (6.32) into (6.31), we find

$$
E_{q m}=-\frac{1}{\pi m} \sum_{n=1}^{\infty} \frac{(2 n-2) ! q^{2 n}}{2^{2 n-2} n !(n-1) !} \int_{0}^{\pi / 2} \sin ^{2 n x} \cos 2 m x d x .
$$

On inserting (6.24) into this, we obtain

$$
E_{q m}=\frac{(-1)^{m+1}}{m} \sum_{n=m}^{\infty} \frac{(2 n) !(2 n-2) ! q^{2 n}}{2^{4 n-1} n !(n-1) !(n+m) !(n-m) !} \cdot
$$

Thus $E_{q m}$ is of order $q^{2 m}$ or of order $k^{m}$ and

Then, for our purposes

$$
\begin{aligned}
& E_{q 1}=\frac{q^{2}}{8}+\frac{q^{4}}{32}+\ldots . \\
& E_{q 2}=-\frac{q^{4}}{256}+\ldots .
\end{aligned}
$$

$$
E(\psi, q)=(2 / \pi) E(q) \psi+\left(\frac{q^{2}}{8}+\frac{q^{4}}{32}\right) \sin 2 \psi-\frac{q^{4}}{256} \sin 4 \psi+\ldots
$$

Finally, inserting (6.28) and (6.37) into (6.10) and (6.12), we have for the $\eta$-integrals $N_{1}$ and $N_{2}$ :

with

$$
\begin{aligned}
& N_{1}=\left(\alpha_{2}^{2}-\alpha_{3}^{2}\right)^{-\frac{1}{2}} \eta_{0}^{3}\left[B_{1} \psi-\frac{1}{4}\left(1+\frac{1}{2} q^{2}\right) \sin 2 \psi+\frac{q^{2}}{64} \sin 4 \psi+\ldots\right], \\
& N_{2}=\left(\alpha_{2}^{2}-\alpha_{3}^{2}\right)^{-\frac{1}{2}} \eta_{0}\left[B_{2} \psi-\frac{q^{2}}{32}\left(4+3 q^{2}\right) \sin 2 \psi+\frac{3 q^{4}}{256} \sin 4 \psi+\ldots\right],
\end{aligned}
$$

$$
\begin{aligned}
& B_{1} \equiv \frac{2 q^{-2}}{\pi}[K(q)-E(q)]=\frac{1}{2}+\frac{3}{8} q^{2}+\frac{15}{128} q^{4}+\ldots . \\
& B_{2} \equiv \frac{2}{\pi} K(q)=1+\frac{1}{4} q^{2}+\frac{9}{64} q^{4}+\ldots
\end{aligned}
$$

Here the terms in $\psi$ are exact. In $N_{2}$ the sines are correct to $O\left(k^{2}\right)$, while in $N_{1}$ they are correct only to $O(k)$; this is as much accuracy as we need for $N_{1}$, however, since it is multiplied by $c^{2}=k p^{2}$ in the first kinetic equation (2.5).

From (6.3) and (3.34) we have

\subsection{The Integral $N_{3}$}

Then since

$$
\left(\alpha_{2}^{2}-\alpha_{3}^{2}\right)^{\frac{1}{2}} N_{3}= \pm \int_{0}^{\eta}\left(1-\eta^{2}\right)^{-1}\left(1-\eta^{2} / \eta_{0}^{2}\right)^{-\frac{1}{2}}\left(1-\eta^{2} / \eta_{2}^{2}\right)^{-\frac{1}{2}} d \eta .
$$

we obtain

$$
\left(1-\eta^{2} / \eta_{2}^{2}\right)^{-\frac{1}{2}}=\sum_{m=0}^{\infty} \frac{(2 m) !}{2^{2 m}(m !)^{2}}\left(\eta / \eta_{2}\right)^{2 m},
$$

where

$$
\left(\alpha_{2}^{2}-\alpha_{3}^{2}\right)^{\frac{1}{2}} N_{3}=\sum_{m=0}^{\infty} \frac{(2 m) ! \eta_{2}^{-2 m}}{2^{2 m}(m !)^{2}} L_{m}
$$

$$
L_{m} \equiv \pm \int_{0}^{\eta}\left(1-\eta^{2}\right)^{-1}\left(1-\eta^{2} / \eta_{0}^{2}\right)^{-\frac{1}{2}} \eta^{2 m} d \eta
$$


With

and

$$
L_{0} \equiv \pm \int_{0}^{\eta}\left(1-\eta^{2}\right)^{-1}\left(1-\eta^{2} / \eta_{0}^{2}\right)^{-\frac{1}{2}} d \eta
$$

$$
\left(1-\eta^{2}\right)^{-1} \eta^{2 m} \equiv\left(1-\eta^{2}\right)^{-1}-\sum_{n=0}^{m-1} \eta^{2 n}, \quad(m \geqq 1),
$$

we then find

$$
L_{m}=L_{0}-\sum_{n=0}^{m-1} L_{1 n}, \quad(m \geqq 1),
$$

where

$$
L_{1 n}= \pm \int_{0}^{\eta} \eta^{2 n}\left(1-\eta^{2} / \eta_{0}^{2}\right)^{-\frac{1}{2}} d \eta
$$

To evaluate $L_{0}$, rewrite (6.46) as

$$
L_{0}= \pm \int_{0}^{\eta} \eta^{-3}\left(\eta^{-2}-1\right)^{-1}\left(\eta^{-2}-\eta_{0}^{-2}\right)^{-\frac{1}{2}} d \eta
$$

and introduce the new variable $\chi$, defined by the equation

$$
\tan \chi=\left(1-\eta_{0}^{2}\right)^{\frac{1}{2}} \tan \psi=|\cos I| \tan \psi
$$

and the requirement that $\chi$ and $\psi$ shall keep in step. I.e., whenever $\psi$ equals a multiple of $\pi / 2, \chi$ shall be equal to $\psi$. Then

and

$$
\csc ^{2} \psi=1+\cos ^{2} I \cot ^{2} \chi
$$

so that

$$
\eta^{-2}=\eta_{0}^{-2} \csc ^{2} \psi=1+\cot ^{2} I \csc ^{2} \chi,
$$

and $\cot \chi d \chi \gtrless 0$ according as $d \eta \gtrless 0$ in (6.50). With $\eta=\eta_{0} \sin \psi$ its integrand becomes

so that

$$
\pm|\tan I| \cot \chi|\tan \chi| d \chi=|\tan I| d \chi
$$

$$
L_{0}=|\tan I| \chi=\eta_{0}\left(1-\eta_{0}^{2}\right)^{-\frac{1}{2}} \chi .
$$

To fit the angle $\chi$ into one's knowledge of the corresponding elliptic motion, note that as $c$ approaches zero, $\eta$ approaches $\sin \theta$. Then, by (6.53),

$$
\chi \rightarrow \sin ^{-1}\left(\frac{\tan \theta}{|\tan I|}\right)=\phi-\Omega,
$$

where $\Omega$ is the right ascension of the node.

To evaluate the integrals $L_{1 n}$, put $\eta=\eta_{0} \sin \psi$ in (6.49). Then

so that

$$
L_{1 n}=\eta_{0}^{2 n+1} \int_{0}^{\psi} \sin ^{2 n} x d x
$$

$$
L_{10}=\eta_{0} \psi \text {. }
$$

To handle the cases $n \geqq 1$, we rewrite (6.22) as

thus finding

$$
\sin ^{2 n} x=\frac{(2 n) !}{2^{2 n}(n !)^{2}}+2^{1-2 n} \sum_{j=1}^{n} \frac{(-1)^{j}(2 n) !}{(n+j) !(n-j) !} \cos 2 j x,
$$

$$
L_{1 n}=\frac{\eta_{0}^{2 n+1}(2 n) ! \psi}{2^{2 n}(n !)^{2}}+\eta_{0}^{2 n+1} 2^{-2 n} \sum_{j=1}^{n} \frac{(-1)^{j}(2 n) ! \sin 2 j \psi}{(n+j) !(n-j) ! j}, \quad(n \geqq 1) .
$$

Insertion of (6.48), (6.59), and (6.61) into (6.44) then yields 


$$
\begin{aligned}
\left(\alpha_{2}^{2}-\alpha_{3}^{2}\right)^{\frac{1}{2}} N_{3}=L_{0} \sum_{m=0}^{\infty} \frac{(2 m) ! \eta_{2}^{-2 m}}{2^{2 m}(m !)^{2}}-\eta_{0} \psi & \sum_{m=1}^{\infty} \frac{(2 m) ! \eta_{2}^{-2 m}}{2^{2 m}(m !)^{2}}-\psi \sum_{m=2}^{\infty} \frac{(2 m) ! \eta_{2}^{-2 m}}{2^{2 m}(m !)^{2}} \sum_{n=1}^{m-1} \frac{\eta_{0}^{2 n+1}(2 n) !}{2^{2 n}(n !)^{2}} \\
& -\sum_{m=2}^{\infty} \frac{(2 m) ! \eta_{2}^{-2 m}}{2^{2 m}(m !)^{2}} \sum_{n=1}^{m-1} \eta_{0}^{2 n+1} 2^{-2 n} \sum_{j=1}^{n} \frac{(-1)^{j}(2 n) ! \sin 2 j \psi}{(n+j) !(n-j) ! j} .
\end{aligned}
$$

If in (6.62) we now use (6.56) and the relation

we find

$$
\left(1-\eta_{2}^{-2}\right)^{-\frac{1}{2}}=\sum_{m=0}^{\infty} \frac{(2 m) ! \eta_{2}^{-2 m}}{2^{2 m}(m !)^{2}}
$$

where

$$
\left(\alpha_{2}^{2}-\alpha_{3}^{2}\right)^{\frac{1}{2}} N_{3}=\eta_{0}\left[\left(1-\eta_{0}^{2}\right)^{-\frac{1}{2}}\left(1-\eta_{2}^{-2}\right)^{-\frac{1}{2}} \chi+B_{3} \psi+\sum_{s=1}^{\infty} B_{3 s} \sin 2 s \psi\right],
$$

and

$$
\begin{aligned}
& B_{3}=1-\left(1-\eta_{2}^{-2}\right)^{-\frac{1}{2}}-\sum_{m=2}^{\infty} \gamma_{m} \eta_{2}^{-2 m}, \\
& \gamma_{m} \equiv \frac{(2 m) !}{2^{2 m}(m !)^{2}} \sum_{n=1}^{m-1} \frac{(2 n) ! \eta_{0}^{2 n}}{2^{2 n}(n !)^{2}}
\end{aligned}
$$

$$
\sum_{s^{\prime}=1}^{\infty} B_{3 s^{\prime}} \sin 2 s^{\prime} \psi=-\sum_{m=2}^{\infty} \frac{(2 m) ! \eta_{2}^{-2 m}}{2^{2 m}(m !)^{2}} \sum_{n=1}^{m-1} \eta_{0}^{2 n} 2^{-2 n} \sum_{j=1}^{n} \frac{(-1)^{j}(2 n) ! \sin 2 j \psi}{(n+j) !(n-j) ! j} .
$$

The easiest way to isolate the coefficients $B_{3 s}$ is to use the orthogonality of the functions $\sin 2 s \psi$. Then

$$
\begin{aligned}
B_{3 s} & =-\sum_{m=s+1}^{\infty} \frac{(2 m) ! \eta_{2}^{-2 m}}{2^{2 m}(m !)^{2}} \sum_{n=s}^{m-1} \frac{\eta_{0}^{2 n} 2^{-2 n}(-1)^{s}(2 n) !}{(n+s) !(n-s) ! s} \\
& =O\left(\eta_{2}^{-2 s-2}\right),
\end{aligned}
$$

so that $B_{31}=O\left(\eta_{2}^{-4}\right)=O\left(k^{2}\right), \quad B_{32}=O\left(\eta_{2}^{-6}\right)=O\left(k^{3}\right)$, etc. Thus, to obtain the sine terms of $N_{3}$ to $O\left(k^{2}\right)$, we need only

$$
B_{31}=\frac{3}{32} \eta_{0}^{2} \eta_{2}^{-4}+\ldots
$$

To test the convergence of the series $\sum_{2}^{\infty} \gamma_{m} \eta_{2}^{-2 m}$, note first that

and hence that

$$
\frac{(2 n) !}{2^{2 n}(n !)^{2}}=\frac{1 \cdot 3 \cdot 5 \ldots(2 n-1)}{2 \cdot 4 \cdot 6 \ldots 2 n} \leqq \frac{1}{2}, \quad(n \geqq 1)
$$

$$
\sum_{n=1}^{m-1} \frac{(2 n) ! \eta_{0}^{2 n+1}}{2^{2 n}(n !)^{2}} \leqq \frac{m-1}{2}
$$

for any orbit, polar or nonpolar. Then, by (6.66), (6.71), and (6.72)

Thus $\gamma_{m}<m$ and

$$
\gamma_{m} \leqq \frac{3}{8}\left(\frac{m-1}{2}\right) \leqq \frac{3}{16}(m-1), \quad(m \geqq 2) .
$$

$$
\sum_{m=2}^{\infty} \gamma_{m} \eta_{2}^{-2 m}<\sum_{m=2}^{\infty} m \eta_{2}^{-2 m}
$$

But

$$
\sum_{2}^{\infty} m \eta_{2}^{-2 m}=\frac{\eta_{2}^{-2}}{\left(1-\eta_{2}^{-2}\right)^{2}}-\eta_{2}^{-2}=\frac{\eta_{2}^{-4}\left(2-\eta_{2}^{-2}\right)}{\left(1-\eta_{2}^{-2}\right)^{2}}
$$

where $\eta_{2}^{-2}=O(k)$. Thus the series $\sum_{2}^{\infty} \gamma_{m} \eta_{2}^{-2 m}$ converges rapidly. 
To test the convergence of the Fourier series $\sum_{s=1}^{\infty} B_{3 s} \sin 2 s \psi$, note that

$$
\left|\sum_{s=1}^{\infty} B_{3 s} \sin 2 s \psi\right| \leqq \sum_{s=1}^{\infty}\left|B_{3 s}\right| \leqq \sum_{m=s+1}^{\infty} \frac{(2 m) ! \eta_{2}^{-2 m}}{2^{2 m}(m !)^{2}} \sum_{n=s}^{m-1} \frac{(2 n) !}{2^{2 n}(n+s) !(n-s) !} .
$$

But

$\frac{(2 n) !}{2^{2 n}(n+s) !(n-s) !}=\frac{(2 n) ! n ! n !}{2^{2 n}(n !)^{2}(n+s) !(n-s) !}=\frac{(2 n) !}{2^{2 n}(n !)^{2}} \frac{(n)(n-1)(n-2) \ldots(n-s+1)}{(n+s)(n+s-1)(n+s-2) \ldots(n+1)} \leqq \frac{(2 n) !}{2^{2 n}(n !)^{2}}<1$, by (6.71). Then

and

$$
\sum_{n=s}^{m-1} \frac{(2 n) !}{2^{2 n}(n+s) !(n-s) !}<m
$$

$$
\left|\sum_{s=1}^{\infty} B_{3 s} \sin 2 s \psi\right|<\sum_{m=s+1}^{\infty} \frac{m(2 m) ! \eta_{2}^{-2 m}}{2^{2 m}(m !)^{2}}<\sum_{m=2}^{\infty} m \eta_{2}^{-2 m}
$$

by (6.71) again. Then, by (6.75)

$$
\left|\sum_{s=1}^{\infty} B_{3 s} \sin 2{ }_{s} \psi\right|<\frac{\eta_{2}^{-4}\left(2-\eta_{2}^{-2}\right)}{\left(1-\eta_{2}^{-2}\right)^{2}} .
$$

Since $\eta_{2}^{-2}=O(k)$, the Fourier series converges rapidly.

Thus all terms in (6.64) remain finite for all $\eta_{0}^{2} \leqq 1$, except the term involving $\chi$, which apparently may become infinite for polar orbits. Note, however, that $N_{3}$ occurs only in eq (2.7) for the right ascension, when it has a factor $\alpha_{3}$, which vanishes for a polar orbit. To see what happens in this case we must investigate the limit of $\alpha_{3} N_{3}$ as $\eta_{0}^{2} \rightarrow 1$. For a polar orbit we have

$$
\alpha_{3} N_{3}=\alpha_{3}\left(\alpha_{2}^{2}-{ }_{3}^{2} \alpha\right)^{-\frac{1}{2}} \eta_{0}\left(1-\eta_{0}^{2}\right)^{-\frac{1}{2}}\left(1-\eta_{2}^{-2}\right)^{-\frac{1}{2}} \chi+O\left(\alpha_{3}\right) .
$$

But $\left|\alpha_{3}\right|\left(\alpha_{2}^{2}-\alpha_{3}^{2}\right)^{-\frac{1}{2}}=\left|\alpha_{3}\right| \alpha_{2}^{-1}$ to $O\left(\alpha_{3}\right)$ and by (4.8) and (4.2)

$$
\left|\alpha_{3}\right| \alpha_{2}^{-1}=\left(1-\eta_{0}^{2}\right)^{\frac{1}{2}}\left(1-\eta_{2}^{-2} \sin ^{2} i_{0}\right)^{\frac{1}{2}}=\left(1-\eta_{0}^{2}\right)^{\frac{1}{2}}\left(1-\eta_{2}^{-2}\right)^{\frac{1}{2}} \text { to } O\left(\alpha_{3}\right) .
$$

Thus to $O\left(\alpha_{3}\right)$

$$
\alpha_{3} N_{3}=\left(\operatorname{sgn} \alpha_{3}\right) \chi \quad \text { (polar orbit). }
$$

If we now use (6.51) to plot $\chi$ versus $\psi$ for various values of $\eta_{0}^{2}$, we obtain figure 1 .

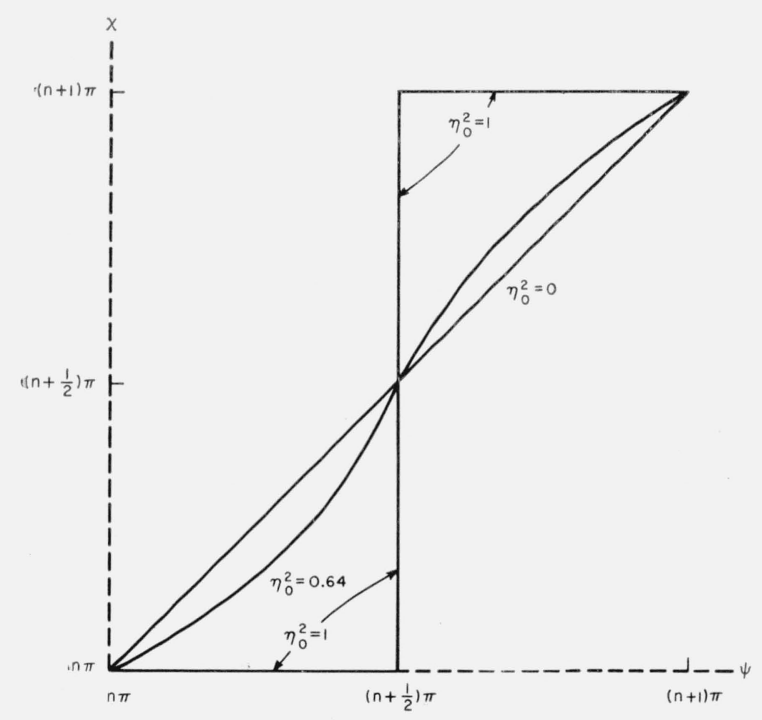

Figure 1. Plot of $\chi$ versus $\psi$ for various values of $\eta_{0}^{2}$. 
The general form of these curves can be checked, as follows. From (6.51)

$$
\tan \chi=\epsilon \tan \psi, \quad \epsilon \equiv\left(1-\eta_{0}^{2}\right)^{\frac{1}{2}} .
$$

To find what happens to a polar orbit we must let $\epsilon$ approach zero.

If we put

$$
\psi=\left(n+\frac{1}{2}\right) \pi+\Delta \psi, \quad \chi=\left(n+\frac{1}{2}\right) \pi+\Delta \chi \quad(n=0,1,2.3, \ldots),
$$

then

$$
\tan \Delta \chi=\epsilon^{-1} \tan \Delta \psi
$$

If we keep $\Delta \psi$ fixed and let $\epsilon \rightarrow 0$, then if $0<\Delta \psi<\pi / 2$, it follows that $\Delta \chi \rightarrow \pi / 2$. If $-\frac{\pi}{2}<\Delta \psi<0$, then $\Delta \chi \rightarrow-\pi / 2$. In a polar orbit, whenever the satellite passes over a pole, $\psi=\left(n+\frac{1}{2}\right) \pi$, with $\dot{\psi}>0$, so that $\chi$ thus jumps by $+\pi$. By (6.80) and (2.7) the right ascension $\phi$ then jumps by $+\pi$ in a direct orbit or by $-\pi$ in a retrograde orbit. These are expected results, which had to be obtained as a partial check of eq (6.64) for $N_{3}$.

\section{Mean Motions}

The purposes of this section are to obtain expressions for the mean motions correct through the first order, to find if we are on the right track, and to obtain exact expressions for the mean frequencies for later use in section 8 in checking the secular terms in the final solution.

If $p_{\rho}, p_{\eta}$, and $p_{\phi}$ are the generalized momenta conjugate to $\rho, \eta$, and $\phi$, then the action variables

$$
\begin{aligned}
& J_{1} \equiv \oint p_{\rho} d \rho=2 \int_{\rho_{1}}^{\rho_{2}} p_{\rho} d \rho, \\
& J_{2} \equiv \oint p_{\eta} d \eta=4 \int_{0}^{\eta_{0}} p_{\eta} d \eta, \\
& J_{3} \equiv \oint p_{\phi} d \phi=\int_{0}^{2 \pi} p_{\phi} d \phi=2 \pi \alpha_{3},
\end{aligned}
$$

are functions of the Jacobi constants $\alpha_{1}, \alpha_{2}, \alpha_{3}$. (Since these $J$ 's occur only in this section, there is no danger of confusion with the coefficients of the zonal harmonics in the expansion of the potential.) The $\alpha$ 's are then functions of these $J$ 's and the mean frequencies [8] are given by

$$
\begin{aligned}
& \text { mean } \rho \text {-frequency }=\nu_{1} \equiv \partial \alpha_{1} / \partial J_{1}, \\
& \text { mean } \eta \text {-frequency }=\nu_{2} \equiv \partial \alpha_{1} / \partial J_{2}, \\
& \text { mean } \phi \text {-frequency }=\nu_{3} \equiv \partial \alpha_{1} / \partial J_{3} .
\end{aligned}
$$

Note that $\nu_{2}$ and $\nu_{3}$ are identical with the usual nodal and sidereal frequencies, but that $\nu_{1}$ is somewhat different from the usual anomalistic frequency.

To compute these frequencies, one may use the system of equations

$$
\sum_{m=1}^{3} \frac{\partial \alpha_{1}}{\partial J_{m}} \frac{\partial J_{m}}{\partial \alpha_{n}}=\frac{\partial \alpha_{1}}{\partial \alpha_{n}}=\delta_{1 n}, \quad(n=1,2,3) .
$$

With the use of (7.4) through (7.6) and the abbreviation

$$
J_{m n} \equiv \partial J_{m} / \partial \alpha_{n}
$$


these equations become

with the solution

$$
\begin{gathered}
\nu_{1} J_{11}+\nu_{2} J_{21}=1, \\
\nu_{1} J_{12}+\nu_{2} J_{22}=0, \\
\nu_{1} J_{13}+\nu_{2} J_{23}+2 \pi \nu_{3}=0, \\
\nu_{1}=J_{22} / \Delta \\
\nu_{2}=-J_{12} / \Delta \\
2 \pi \nu_{3}=-\nu_{1} J_{13}-\nu_{2} J_{23} \\
\Delta \equiv J_{11} J_{22}-J_{12} J_{21}
\end{gathered}
$$

With the use of eqs (13), (53), and (55) of [1] and sections (5) and (6) of the present paper we find

$$
\begin{aligned}
& J_{11}=2 R_{1}\left(\rho_{2}\right)=2 \pi\left(-2 \alpha_{1}\right)^{-\frac{1}{2}}\left(a+b_{1}+A_{1}\right), \\
& J_{12}=-2 \alpha_{2} R_{2}\left(\rho_{2}\right)=-2 \pi \alpha_{2}\left(-2 \alpha_{1}\right)^{-\frac{1}{2}} A_{2}, \\
& J_{13}=2 c^{2} \alpha_{3} R_{3}\left(\rho_{2}\right)=2 \pi c^{2} \alpha_{3}\left(-2 \alpha_{1}\right)^{-\frac{1}{2}} A_{3}, \\
& J_{21}=4 c^{2} N_{1}\left(\eta_{0}\right)=2 \pi c^{2}\left(\alpha_{2}^{2}-\alpha_{3}^{2}\right)^{-\frac{1}{2}} \eta_{0}^{3} B_{1} \\
& J_{22}=4 \alpha_{2} N_{2}\left(\eta_{0}\right)=2 \pi \alpha_{2}\left(\alpha_{2}^{2}-\alpha_{3}^{2}\right)^{-\frac{1}{2}} \eta_{0} B_{2}, \\
& J_{23}=-4 \alpha_{3} N_{3}\left(\eta_{0}\right)=-2 \pi \alpha_{3}\left(\alpha_{2}^{2}-\alpha_{3}^{2}\right)^{-\frac{1}{2}} \eta_{0}\left[B_{3}+\left(1-\eta_{0}^{2}\right)^{-\frac{1}{2}}\left(1-\eta_{2}^{-2}\right)^{-\frac{1}{2}}\right] .
\end{aligned}
$$

Then

$$
\begin{aligned}
& 2 \pi \nu_{1}=\left(-2 \alpha_{1}\right)^{\frac{1}{2}}\left[a+b_{1}+A_{1}+c^{2} \eta_{0}^{2} A_{2} B_{1} B_{2}^{-1}\right]^{-1} \\
& 2 \pi \nu_{2}=\left(\alpha_{2}^{2}-\alpha_{3}^{2}\right)^{\frac{1}{2}} \eta_{0}^{-1} A_{2} B_{2}^{-1}\left[a+b_{1}+A_{1}+c^{2} \eta_{0}^{2} A_{2} B_{1} B_{2}^{-1}\right]^{-1} .
\end{aligned}
$$

The above results are all exact, for the potential (2.3), and we shall refer to them again in section 8 .

For comparison with other theories it is desirable to express these mean frequencies as power series in the oblateness parameter. For this purpose it is convenient to use the orbital elements $a_{0}, e_{0}$, and $i_{0}$, and since we shall carry the series only through the first power we may replace $k_{0} \equiv c^{2} / p_{0}^{2}$ by $k \equiv c^{2} / p^{2}$.

With use of the relations in sections 3 and 4 , the above equations then become

where $n_{0}$ is given by

$$
\begin{aligned}
& 2 \pi \nu_{1}=n_{0}+O\left(k^{2}\right) \\
& 2 \pi \nu_{2}=n_{0}\left[1+\frac{3}{4} k\left(5 \cos ^{2} i_{0}-1\right)\right]+O\left(k^{2}\right),
\end{aligned}
$$

Similarly

$$
\mu=n_{0}^{2} a_{0}^{3} .
$$

which together with (7.14), (7.24), and (7.25) lead to

$$
2 \pi \nu_{3} / n_{0}=\left(\operatorname{sgn} \alpha_{3}\right)\left[1+\frac{3}{4} k\left(5 \cos ^{2} i_{0}-1\right)\right]-\frac{3}{2} k \cos i_{0}+O\left(k^{2}\right) .
$$

Here $\operatorname{sgn} \alpha_{3}= \pm 1$ accordingly as the orbit is direct or retrograde, respectively.

To avoid any use of the concept of an osculating ellipse, we may define the mean motions as follows. We say that the ascending node exists only when the satellite is over the equator, travelling north. Let $\Omega$ be its right ascension at such a time. We then define the mean 
motion $\bar{\Omega}$ of the node relative to $O X$ by

$$
\overline{\dot{\Omega}} \equiv \lim _{t_{i} \rightarrow \infty} \frac{\Omega_{i}-\Omega_{7}}{t_{i}-t_{0}},
$$

where $\Omega_{0}$ and $t_{0}$ are the values of $\Omega$ and $t$ at some (ascending) node and $\Omega_{i}$ and $t_{i}$ are their values $i$ nodes later. Since the present system is of the conditionally periodic Staeckel type, it follows, after some fairly close reasoning that I shall here omit, that

$$
\overline{\dot{\Omega}}=2 \pi\left(\left|\nu_{3}\right|-\nu_{2}\right) \operatorname{sgn} \alpha_{3} .
$$

We also say that $\rho$-perigee exists when, and only when, $\rho=\rho_{1}$ and we let $\Phi$ be its right ascension at such a time. Then the mean motion of $\rho$-perigee relative to $O X$ must be equal to the mean motion $\overline{\dot{\Phi}}$ of its equatorial projection relative to $O X$ and

$$
\overline{\dot{\Phi}} \equiv \lim _{t_{i} \rightarrow \infty} \frac{\Phi_{i}-\Phi_{0}}{t_{i}-t_{0}}
$$

Here $\Phi_{0}$ and $t_{0}$ are the values of $\Phi$ and $t$ at some $\rho$-perigee and $\Phi_{i}$ and $t_{i}$ are their values $i$ $\rho$-perigees later. Then, again omitting the proof, we have

$$
\overline{\dot{\Phi}}=2 \pi\left(\left|\nu_{3}\right|-\nu_{1}\right) \operatorname{sgn} \alpha_{3},
$$

Now let $\omega$ be the arc on the celestial sphere from an ascending node (when it exists) to the next $\rho$-perigee. Then, since the mean relative motion of these points must be equal to the mean relative motion of their projections in the equatorial plane, we find that the mean motion $\overline{\dot{\omega}}$ of $\rho$-perigee relative to the node is given by

$$
\begin{aligned}
\overline{\dot{\omega}} & =(\overline{\dot{\Phi}}-\overline{\dot{\Omega}}) \operatorname{sgn} \alpha_{3}, \\
& =2 \pi\left(\nu_{2}-\nu_{1}\right),
\end{aligned}
$$

with use of (7.31) and (7.33), for any orbit, direct or retrograde.

From (7.31), (7.25), and (7.29) it follows that

$$
\overline{\dot{\Omega}}=-\frac{3}{2} k n_{0} \cos i_{0}+O\left(k^{2}\right)
$$

and from $(7.35),(7.24)$, and (7.25) that

$$
\overline{\dot{\omega}}=\frac{3}{4} k n_{0}\left(5 \cos ^{2} i_{0}-1\right)+O\left(k^{2}\right)
$$

Here $n_{0} / 2 \pi$ is the frequency in an elliptic orbit with the same total energy. Equation (7.36) agrees with results found by many other authors, as does (7.37) when $\dot{\omega}$ is the mean motion of $r$-perigee relative to the node. By (2.1) and (2.2), however, $r^{2}=\rho^{2}+k p^{2}\left(1-\eta^{2}\right)$, so that $r$ and $\rho$ differ by a variable quantity of $O(k)$. It is thus a little surprising that the mean motions of $\rho$-perigee and of $r$-perigee relative to the node should be equal through $O(k)$. This means that the mean $\rho$-frequency is equal to the mean $r$-frequency to this order.

\section{Solution of the Kinetic Equations}

Before solving the kinetic equations (2.5) through (2.7) it is convenient to have several relations connecting the uniformising variables $E$ and $v$. From (5.12) we obtain 


$$
\cos v=\frac{\cos E-e}{1-e \cos E}
$$

The requirements that $d v / d t>0, d E / d t>0$ for all $t$ lead to the result that $d v / d E>0$ for all $t$. Because of this result, (8.1a) leads to

$$
\sin v=+\frac{\left(1-e^{2}\right)^{\frac{1}{2}} \sin E}{1-e \cos E}
$$

without ambiguity in sign. For a given value of $E$, eqs $(8.1 \mathrm{a}, \mathrm{b})$ determine $v$ modulo $2 \pi$. On imposing the further requirement that $v$ shall always equal $E$ whenever the latter is a multiple of $\pi$, we find that $E$ determines $v$ completely. Two other relations are often useful, viz,

and

$$
\tan \frac{v}{2}=\left(\frac{1+e}{1-e}\right)^{\frac{1}{2}} \tan \frac{E}{2}
$$

$$
\tan \frac{v-E}{2}=\frac{\gamma \sin E}{1-\gamma \cos E}, \quad \gamma \equiv e^{-1}\left[1-\left(1-e^{2}\right)^{\frac{1}{2}}\right]<1
$$

Before beginning the solution of the kinetic equations it is desirable to assemble the results already obtained. By (2.5), (2.6), (5.1), (5.2), (5.30) through (5.33), (5.6), (5.28), (5.35) through (5.40), (6.1), (6.2), (6.6), and (6.38) through (6.41), the equations for $\rho$ and $\eta$ are

$$
\begin{aligned}
t+\beta_{1} & =\left(-2 \alpha_{1}\right)^{-\frac{1}{2}}\left[b_{1} E+a(E-e \sin E)+A_{1} v+A_{11} \sin v+A_{12} \sin 2 v\right] \\
& +c^{2}\left(\alpha_{2}^{2}-\alpha_{3}^{2}\right)^{-\frac{1}{2}} \eta_{0}^{3}\left[B_{1} \psi-\frac{1}{8}\left(2+q^{2}\right) \sin 2 \psi+\frac{q^{2}}{64} \sin 4 \psi\right]+\text { periodic terms of } O\left(k^{3}\right),
\end{aligned}
$$

$\beta_{2} / \alpha_{2}=-\left(-2 \alpha_{1}\right)^{-\frac{1}{2}}\left[A_{2} v+A_{21} \sin v+A_{22} \sin 2 v+A_{23} \sin 3 v+A_{24} \sin 4 v\right]$

Here

$$
+\left(\alpha_{2}^{2}-\alpha_{3}^{2}\right)^{-\frac{1}{2}} \eta_{0}\left[B_{2} \psi-\frac{q^{2}}{32}\left(4+3 q^{2}\right) \sin 2 \psi+\frac{3 q^{4}}{256} \sin 4 \psi\right]+\text { periodic terms of } O\left(k^{3}\right) .
$$

$$
\begin{aligned}
\rho=a(1-e \cos E) & =a\left(1-e^{2}\right)(1+e \cos v)^{-1}, \\
\eta & =\eta_{0} \sin \psi,
\end{aligned}
$$

with $E$ and $v$ connected by any of the eqs (8.1).

$$
\begin{aligned}
A_{1} & =\left(1-e^{2}\right)^{\frac{1}{2}} p \sum_{n=2}^{\infty}\left(b_{2} / p\right)^{n} P_{n}\left(b_{1} / b_{2}\right) R_{n-2}\left[\left(1-e^{2}\right)^{\frac{1}{2}}\right]=O(k), \\
A_{2} & =\left(1-e^{2}\right)^{\frac{1}{2}} p^{-1} \sum_{n=0}^{\infty}\left(b_{2} / p\right)^{n} P_{n}\left(b_{1} / b_{2}\right) R_{n}\left[\left(1-e^{2}\right)^{\frac{1}{2}}\right]=O\left(k^{0}\right), \\
A_{11} & =\frac{3}{4}\left(1-e^{2}\right)^{\frac{1}{2}} p^{-3} e\left(-2 b_{1} b_{2}^{2} p+b_{2}^{4}\right)=O\left(k^{2}\right), \\
A_{12} & =\frac{3}{32}\left(1-e^{2}\right)^{\frac{1}{2}} p^{-3} b_{2}^{4} e^{2}=O\left(k^{2}\right), \\
B_{1} & =\frac{2}{\pi q^{2}}[K(q)-E(q)]=O\left(k^{0}\right), \\
B_{2} & =\frac{2}{\pi} K(q)=O\left(k^{0}\right), \\
q & =\eta_{0} / \eta_{2}=O\left(k^{\frac{1}{2}}\right) .
\end{aligned}
$$

$K(q)$ and $E(q)$ are the complete elliptic integrals of the first and second kinds. 


$$
\begin{aligned}
& A_{21}=\left(1-e^{2}\right)^{\frac{1}{2}} e p^{-5}\left[-\frac{1}{2} A p^{3}+\left(\frac{3}{4} A^{2}-B\right) p^{2}+\frac{9}{4} A B p\left(1+\frac{e^{2}}{4}\right)+\frac{3}{8} B^{2}\left(4+3 e^{2}\right)\right]=O(k), \\
& A_{22}=\left(1-e^{2}\right)^{\frac{1}{2}} p^{-5}\left[\left(\frac{3}{8} A^{2}-\frac{1}{2} B\right) \frac{e^{2}}{4} p^{2}+\frac{9}{16} A B e^{2} p+\frac{3}{8} B^{2}\left(\frac{3}{2} e^{2}+\frac{1}{4} e^{4}\right)\right]=O(k), \\
& A_{23}=\left(1-e^{2}\right)^{\frac{1}{2}} p^{-5} e\left[\frac{A B e^{2} p}{16}+\frac{B^{2} e^{2}}{8}\right]=O\left(k^{2}\right), \\
& A_{21}=\frac{3}{256}\left(1-e^{2}\right)^{\frac{1}{2}} p^{-5} B^{2} e^{4}=O\left(k^{2}\right) .
\end{aligned}
$$

In the above equations $c^{2}=r_{e}^{2} J_{2}$ is regarded as known, as are the orbital elements $a, e$, $\eta_{0}, \beta_{1}$, and $\beta_{2}$. Then $p=a\left(1-e^{2}\right)$ and $\eta_{2}, A, B, \alpha_{1}, \alpha_{2}$, and $\alpha_{3}$ are given by section 4 . Also $b_{1}=-\frac{1}{2} A, b_{2}^{2}=B, \quad R_{m}(x)=x^{m} P_{m}(1 / x)$, with $|x|<1$.

To solve (8.2) and (8.3), place

$$
E=E_{s}+E_{p}, \quad v=v_{s}+v_{p}, \quad \psi=\psi_{s}+\psi_{p},
$$

where the subscript $s$ means "secular" and the subscript $p$ "periodic." Then if $\rho$ goes through $N_{1}$ cycles in time $T_{1}$ and if $\eta$ goes through $N_{2}$ cycles in time $T_{2}$,

$$
\begin{aligned}
& \overline{\dot{E}}=\overline{\dot{v}}=\dot{E}_{s}=\dot{v}_{s}=\lim _{T_{1} \rightarrow \infty} \frac{2 \pi N_{1}}{T_{1}}=2 \pi \nu_{1} \\
& \overline{\dot{\psi}}=\dot{\psi}_{s}=\lim _{T_{2} \rightarrow \infty} \frac{2 \pi N_{2}}{T_{2}}=2 \pi \nu_{2} .
\end{aligned}
$$

Since we have already obtained exact expressions in section 7 for $\nu_{1}$ and $\nu_{2}$, it is clear that we can obtain the secular terms exactly. We shall also obtain the periodic terms through $O\left(k^{2}\right)$.

By (8.18) we can write

$$
E_{s}=v_{s}=M_{s},
$$

where $M_{s}$ will play the role of the secular part of a mean anomaly.

Then

$$
E=M_{s}+E_{p}, \quad v=M_{s}+v_{p}, \quad \psi=\psi_{s}+\psi_{p} .
$$

We may obtain the secular solution of (8.2) and (8.3) independently of section 7 , by dropping all the sines in those equations, placing $E=v=M_{s}$ and $\psi=\psi_{s}$, and solving the resulting equations for $M_{s}$ and $\psi_{s}$. The resulting equations are

$$
\begin{gathered}
\left(-2 \alpha_{1}\right)^{-\frac{1}{2}}\left(a+b_{1}+A_{1}\right) M_{s}+c^{2}\left(\alpha_{2}^{2}-\alpha_{3}^{2}\right)^{-\frac{1}{2}} \eta_{0}^{3} B_{1} \psi_{s}=t+\beta_{1}, \\
-\left(-2 \alpha_{1}\right)^{-\frac{1}{2}} A_{2} M_{s}+\left(\alpha_{2}^{2}-\alpha_{3}^{2}\right)^{-\frac{1}{2}} \eta_{0} B_{2} \psi_{s}=\beta_{2} / \alpha_{2},
\end{gathered}
$$

with the following solution.

$$
\begin{gathered}
\text { 8.1. Secular Solution } \\
M_{s}=\left(-2 \alpha_{1}\right)^{\frac{1}{2}} \frac{B_{2}\left(t+\beta_{1}\right)-c^{2} \beta_{2} \alpha_{2}^{-1} \eta_{0}^{2} B_{1}}{\left(a+b_{1}+A_{1}\right) B_{2}+c^{2} \eta_{0}^{2} A_{2} B_{1}} \\
\psi_{s}=\left(\alpha_{2}^{2}-\alpha_{3}^{2}\right)^{\frac{1}{2}} \eta_{0}^{-1} \frac{A_{2}\left(t+\beta_{1}\right)+\beta_{2} \alpha_{2}^{-1}\left(a+b_{1}+A_{1}\right)}{\left(a+b_{1}+A_{1}\right) B_{2}+c^{2} \eta_{0}^{2} A_{2} B_{1}} .
\end{gathered}
$$

Comparison of these results with (7.22) and (7.23) shows that $\dot{M}_{s}=2 \pi \nu_{1}$ and $\dot{\psi}_{s}=2 \pi \nu_{2}$, as expected. We may now rewrite these expressions more conveniently as

$$
M_{s}=2 \pi \nu_{1}\left(t+\beta_{1}-c^{2} \beta_{2} \alpha_{2}^{-1} \eta_{0}^{2} B_{1} B_{2}^{-1}\right),
$$




$$
\psi_{s}=2 \pi \nu_{2}\left[t+\beta_{1}+\beta_{2} \alpha_{2}^{-1}\left(a+b_{1}+A_{1}\right) A_{2}^{-1}\right] .
$$

As a check, note that to $O\left(k^{0}\right), 2 \pi \nu_{1}=2 \pi \nu_{2}=n_{0}$, so that

$$
\begin{gathered}
M_{s}=n_{0}\left(t+\beta_{1}\right)+O(k), \\
\psi_{s}=n_{0}\left(t+\beta_{1}\right)+\frac{n_{0} a \beta_{2}}{\alpha_{2} A_{2}}+O(k) .
\end{gathered}
$$

But, to order $k^{0}, \quad \alpha_{2}=(\mu p)^{\frac{1}{2}}$ and $A_{2}=\left(1-e^{2}\right)^{\frac{1}{2}} p^{-1}$, so that $n_{0} a\left(\alpha_{2} A_{2}\right)^{-1}=n_{0} a p\left[\mu p\left(1-e^{2}\right)\right]^{-\frac{1}{2}}=1$, since $n_{0}^{2} a^{3}=\mu$ to $O\left(k^{0}\right)$.

Thus

$$
\psi_{s}=M_{s}+\beta_{z}+O(k)
$$

as is to be expected, with $\beta_{2}$ replacing $\omega$.

As a later aid in reducing the solution for the periodic terms to Kepler's equation, it is here convenient to rewrite (8.22), by transposition of some of its terms:

$$
t+\beta_{1}-\left(-2 \alpha_{1}\right)^{-\frac{1}{2}}\left(a+b_{1}\right) M_{s}=\left(-2 \alpha_{1}\right)^{-\frac{1}{2}} A_{1} M_{s}+c^{2}\left(\alpha_{2}^{2}-\alpha_{3}^{2}\right)^{-\frac{1}{2}} \eta_{0}^{3} B_{1} \psi_{s} .
$$

\subsection{Periodic Terms}

We shall put, successively,

$$
\begin{array}{lll}
E_{p}=E_{0}, & v_{p}=v_{0}, & \psi_{p}=\psi_{0} ; \\
E_{p}=E_{0}+E_{1}, & v_{p}=v_{0}+v_{1}, & \psi_{p}=\psi_{0}+\psi_{1} ; \\
E_{p}=E_{0}+E_{1}+E_{2}, & v_{p}=v_{0}+v_{1}+v_{2}, & \psi_{p}=\psi_{0}+\psi_{1}+\psi_{2} .
\end{array}
$$

In step 0, we retain in the equations (8.2) and (8.3) only those periodic terms which are of $\left(O k^{0}\right)$, viz, $\sin E$. In step 1, we retain in these equations all periodic terms of order $k^{0}$ or $k$, but none of higher order. In step 2, we retain in the equations all periodic terms of order $k^{0}$, $k$, or $k^{2}$, but none of higher order. In carrying out each step, bowever, we shall suppose that each quantity involved is calculated to such an accuracy that the error is of order $k^{3}$. Then, effectively, $E_{0}, v_{0}$, and $\psi_{0}$ will all contain terms of order $k$ and $k^{2}$, as well as terms of order $k^{0}$. $E_{1}, v_{1}$, and $\psi_{1}$ will contain no terms of order $k^{0}$, but will contain terms of order $k$ and $k^{2}$. $E_{2}$, $v_{2}$, and $\psi_{2}$ will be of order $k^{2}$. Such a procedure will greatly simplify the resulting equations.

\subsection{The Periodic Contributions $E_{0}, v_{0}$, and $\psi_{0}$}

On placing $E=M_{s}+E_{0}, v=M_{s}+v_{0}$, and $\psi=\psi_{s}+\psi_{0}$ in (8.2) and (8.3) and retaining only the term $\sin E$ of the periodic terms, we find

$$
\begin{gathered}
t+\beta_{1}=\left(-2 \alpha_{1}\right)^{-\frac{1}{2}}\left[\left(a+b_{1}\right)\left(M_{s}+E_{0}\right)-a e \sin \left(M_{s}+E_{0}\right)+A_{1} M_{s}\right]+c^{2}\left(\alpha_{2}^{2}-\alpha_{3}^{2}\right)^{-\frac{1}{2}} \eta_{0}^{3} B_{1} \psi_{s}, \\
\beta_{2} / \alpha_{2}=-\left(-2 \alpha_{1}\right)^{-\frac{1}{2}} A_{2}\left(M_{s}+v_{0}\right)+\left(\alpha_{2}^{2}-\alpha_{3}^{2}\right)^{-\frac{1}{2}} \eta_{0} B_{2}\left(\psi_{s}+\psi_{0}\right) .
\end{gathered}
$$

On subtracting (8.28) from (8.29) and dividing the resulting equation by $\left(a+b_{1}\right)\left(-2 \alpha_{1}\right)^{-\frac{1}{2}}$, we find

$$
M_{s}+E_{0}-e^{\prime} \sin \left(M_{s}+E_{0}\right)=M_{s},
$$

where

$$
e^{\prime} \equiv \frac{a e}{a+b_{1}}<1
$$

since $b_{1}>0$. Equation (8.31) is Kepler's equation for $M_{s}+E_{0}$, with an effective eccentricity $e^{\prime}$. Let us suppose it to be solved by the most appropriate method, which will depend on the value of $e^{\prime}$. We then have $M_{s}+E_{0}$ and can then find $v=M_{s}+v_{0}$ by use of eqs (8.1). 
On subtracting (8.23) from (8.30) we then obtain $\psi_{0}$ as a function of $v_{0}$ :

$$
\psi_{0}=\left(-2 \alpha_{1}\right)^{-\frac{1}{2}}\left(\alpha_{2}^{2}-\alpha_{3}^{2}\right)^{\frac{1}{2}} \eta_{0}^{-1} A_{2} B_{2}^{-1} v_{0} .
$$

Here the coefficient $\psi_{0} / v_{0}$ is unity to $O\left(k^{0}\right)$, but to follow the procedure outlined above we must not make such an approximation. Instead we must calculate it so accurately that the error is of order $k^{3}$.

\subsection{The Periodic Contributions $E_{1}, v_{1}$, and $\psi_{1}$}

Now, knowing $M_{s}, \psi_{s}, E_{0}, v_{0}$, and $\psi_{0}$, we place $E=M_{s}+E_{0}+E_{1}, v=M_{s}+v_{0}+v_{1}$, and $\psi=$ $\psi_{s}+\psi_{0}+\psi_{1}$ into eqs (8.2) and (8.3), discarding only the periodic terms of order $k^{2}$. Then

$$
\begin{aligned}
t+\beta_{1}=\left(-2 \alpha_{1}\right)^{-\frac{1}{2}}\left[\left(a+b_{1}\right)\left(M_{s}+E_{0}+E_{1}\right)-a e\right. & \left.\sin \left(M_{s}+E_{0}+E_{1}\right)+A_{1}\left(M_{s}+v_{0}\right)\right] \\
& +c^{2}\left(\alpha_{2}^{2}-\alpha_{3}^{2}\right)^{-\frac{1}{2}} \eta_{0}^{3}\left[B_{1}\left(\psi_{s}+\psi_{0}\right)-\frac{1}{4} \sin \left(2 \psi_{s}+2 \psi_{0}\right)\right],
\end{aligned}
$$

$\beta_{2} / \alpha_{2}=-\left(-2 \alpha_{1}\right)^{-\frac{1}{2}}\left[A_{2}\left(M_{s}+v_{0}+v_{1}\right)+A_{21} \sin \left(M_{s}+v_{0}\right)+A_{22} \sin \left(2 M_{s}+2 v_{0}\right)\right]$

$$
+\left(\alpha_{2}^{2}-\alpha_{3}^{2}\right)^{-\frac{1}{2}} \eta_{0}\left[B_{2}\left(\psi_{s}+\psi_{0}+\psi_{1}\right)-\frac{q^{2}}{8} \sin \left(2 \psi_{s}+2 \psi_{0}\right)\right] .
$$

Subtraction of (8.28) from (8.34) and division of the result by $\left(a+b_{1}\right)\left(-2 \alpha_{1}\right)^{-\frac{1}{2}}$ now gives a Kepler equation for $M_{s}+E_{0}+E_{1}$ :

where

$$
M_{s}+E_{0}+E_{1}-e^{\prime} \sin \left(M_{s}+E_{0}+E_{1}\right)=M_{s}+M_{1},
$$

$$
M_{1} \equiv\left(a+b_{1}\right)^{-1}\left[-\left(A_{1}+c^{2} \eta_{0}^{2} A_{2} B_{1} B_{2}^{-1}\right) v_{0}+\frac{c^{2}}{4}\left(-2 \alpha_{1}\right)^{\frac{1}{2}}\left(\alpha_{2}^{2}-\alpha_{3}^{2}\right)^{-\frac{1}{2}} \eta_{0}^{3} \sin \left(2 \psi_{s}+2 \psi_{0}\right)\right] .
$$

Here we have used (8.33) to combine terms in $v_{0}$ and $\psi_{0}$. The quantity $M_{s}+M_{1}$ is then a mean anomaly whose secular part is exact and whose periodic part is correct through order $k$. It has no periodic part of order $k^{0}$; this is characteristic of a mean anomaly.

It is not necessary to solve the Kepler equation all over again. If in (8.36) we put

$$
\sin \left(M_{s}+E_{0}+E_{1}\right)=\left(1-\frac{1}{2} E_{1}^{2}\right) \sin \left(M_{s}+E_{0}\right)+E_{1} \cos \left(M_{s}+E_{0}\right)+O\left(E_{1}^{3}\right),
$$

the error is of order $k^{3}$. Then (8.36) and (8.38) yield a quadratic equation for $E_{1}$, whose solution through terms of $O\left(k^{2}\right)$ is given by

$$
E_{1}=\frac{M_{1}}{1-e^{\prime} \cos \left(M_{s}+E_{0}\right)}-\frac{e^{\prime}}{2} \frac{M_{1}^{2} \sin \left(M_{s}+E_{0}\right)}{\left[1-e^{\prime} \cos \left(M_{s}+E_{0}\right)\right]^{3}} .
$$

To find $v_{1}$ insert $v=M_{s}+v_{0}+v_{1}$ and $E=M_{s}+E_{0}+E_{1}$ into eqs (8.1) and solve for $v_{1}$.

On subtracting (8.23) from (8.35) and eliminating terms in $v_{0}$ and $\psi_{0}$ by use of (8.33), we then find

$$
\begin{gathered}
\psi_{1}=\left(-2 \alpha_{1}\right)^{-\frac{1}{2}}\left(\alpha_{2}^{2}-\alpha_{3}^{2}\right)^{\frac{1}{2}} \eta_{0}^{-1} B_{2}^{-1}\left[A_{2} v_{1}+A_{21} \sin \left(M_{s}+v_{0}\right)+A_{22} \sin \left(2 M_{s}+2 v_{0}\right)\right] \\
+\frac{q^{2}}{8} B_{2}^{-1} \sin \left(2 \psi_{s}+2 \psi_{0}\right)
\end{gathered}
$$

(Note that the elimination of $v_{0}$ and $\psi_{0}$ would not have been possible if they had been carried only through order $k^{0}$.)

\subsection{The Periodic Contributions $E_{2}, v_{2}$, and $\psi_{2}$}

Finally, knowing $M_{s}, \psi_{s}, E_{0}, v_{0}, \psi_{0}, E_{1}, v_{1}$, and $\psi_{1}$, we place $E=M_{s}+E_{0}+E_{1}+E_{2}, \quad v=M_{s}$ $+v_{0}+v_{1}+v_{2}$, and $\psi=\psi_{s}+\psi_{0}+\psi_{1}+\psi_{2}$ in (8.2) and (8.3), discarding only the periodic terms of order greater than $k^{2}$. The equations become 
$t+\beta_{1}=\left(-2 \alpha_{1}\right)^{-\frac{1}{2}}\left[\left(a+b_{1}\right)\left(M_{s}+E_{0}+E_{1}+E_{2}\right)-a e \sin \left(M_{s}+E_{0}+E_{1}+E_{2}\right)+A_{1}\left(M_{s}+v_{0}+v_{1}\right)\right.$

$\left.+A_{11} \sin \left(M_{s}+v_{0}\right)+A_{12} \sin \left(2 M_{s}+2 v_{0}\right)\right]+c^{2}\left(\alpha_{2}^{2}-\alpha_{3}^{2}\right)^{-\frac{1}{2}} \eta_{0}^{3}\left[B_{1}\left(\psi_{s}+\psi_{0}+\psi_{1}\right)\right.$

$$
\left.-\frac{1}{8}\left(2+q^{2}\right) \sin \left(2 \psi_{s}+2 \psi_{0}+2 \psi_{1}\right)+\frac{q^{2}}{64} \sin \left(4 \psi_{s}+4 \psi_{0}\right)\right]
$$

$\beta_{2} / \alpha_{2}=-\left(-2 \alpha_{1}\right)^{-\frac{1}{3}}\left[A_{2}\left(M_{s}+v_{0}+v_{1}+v_{2}\right)+A_{21} \sin \left(M_{s}+v_{0}+v_{1}\right)+A_{22} \sin \left(2 M_{s}+2 v_{0}+2 v_{1}\right)\right.$

$$
\begin{array}{r}
\left.+A_{23} \sin \left(3 M_{s}+3 v_{0}\right)+A_{24} \sin \left(4 M_{s}+4 v_{0}\right)\right]+\left(\alpha_{2}^{2}-\alpha_{3}^{2}\right)^{-\frac{1}{2}} \eta_{0}\left[B_{2}\left(\psi_{s}+\psi_{0}+\psi_{1}+\psi_{2}\right)\right. \\
\left.-\frac{q^{2}}{32}\left(4+3 q^{2}\right) \sin \left(2 \psi_{s}+2 \psi_{0}+2 \psi_{1}\right)+\frac{3}{256} q^{4} \sin \left(4 \psi_{s}+4 \psi_{0}\right)\right] .
\end{array}
$$

On subtracting (8.34) from (8.41) and discarding periodic terms of order $k^{3}$ or higher, we find $0=\left(-2 \alpha_{1}\right)^{-\frac{1}{2}}\left[\left(a+b_{1}\right) E_{2}-a e E_{2} \cos \left(M_{s}+E_{0}+E_{1}\right)+A_{1} v_{1}+A_{11} \sin \left(M_{s}+v_{0}\right)\right.$

$$
\begin{aligned}
\left.+A_{12} \sin \left(2 M_{s}+2 v_{0}\right)\right]+c^{2}\left(\alpha_{2}^{2}-\alpha_{3}^{2}\right)^{-\frac{1}{2}} \eta_{0}^{3} & {\left[B_{1} \psi_{1}-\frac{1}{2} \psi_{1} \cos \left(2 \psi_{s}+2 \psi_{0}\right)\right.} \\
- & \left.\frac{q^{2}}{8} \sin \left(2 \psi_{s}+2 \psi_{0}\right)+\frac{q^{2}}{64} \sin \left(4 \psi_{s}+4 \psi_{0}\right)\right] .
\end{aligned}
$$

Then

$$
E_{2}=\frac{M_{2}}{1-e^{\prime} \cos \left(M_{s}+E_{0}+E_{1}\right)},
$$

where

$$
\begin{aligned}
& M_{2} \equiv-\left(a+b_{1}\right)^{-1}\left[A_{1} v_{1}+A_{11} \sin \left(M_{s}+v_{0}\right)+A_{12} \sin \left(2 M_{s}+2 v_{0}\right)\right. \\
& \left.+c^{2}\left(-2 \alpha_{1}\right)^{\frac{1}{2}}\left(\alpha_{2}^{2}-\alpha_{3}^{2}\right)^{-\frac{1}{2}} \eta_{0}^{3}\left\{B_{1} \psi_{1}-\frac{1}{2} \psi_{1} \cos \left(2 \psi_{s}+2 \psi_{0}\right)-\frac{q^{2}}{8} \sin \left(2 \psi_{s}+2 \psi_{0}\right)+\frac{q^{2}}{64} \sin \left(4 \psi_{s}+4 \psi_{0}\right)\right\}\right] .
\end{aligned}
$$

It is easy to show that, to order $k^{2}$.

$$
M_{s}+E_{0}+E_{1}+E_{2}-e^{\prime} \sin \left(M_{s}+E_{0}+E_{1}+E_{2}\right)=M_{s}+M_{1}+M_{2},
$$

so that $M_{2}$ is the second-order periodic term of a total mean anomaly

$$
M=M_{s}+M_{1}+M_{2}+\ldots
$$

corresponding to the effective eccentricity $e^{\prime}$.

To find $v_{2}$ insert $v=M_{s}+v_{0}+v_{1}+v_{2}$ and $E=M_{s}+E_{0}+E_{1}+E_{2}$ into eqs (8.1) and solve for $v_{2}$.

To find $\psi_{2}$, subtract (8.35) from (8.42) and discard periodic terms of order $k^{3}$ or higher. The result is

$$
\begin{aligned}
& \psi_{2}=\left(-2 \alpha_{1}\right)^{-\frac{1}{2}}\left(\alpha_{2}^{2}-\alpha_{3}^{2}\right)^{\frac{1}{2}} \eta_{0}^{-1} B_{2}^{-1}\left[A_{2} v_{2}+A_{21} v_{1} \cos \left(M_{s}+v_{0}\right)+2 A_{22} v_{1} \cos \left(2 M_{s}+2 v_{0}\right)+A_{23} \sin \left(3 M_{s}+3 v_{0}\right)\right. \\
& \left.+A_{24} \sin \left(4 M_{s}+4 v_{0}\right)\right]+\frac{q^{2}}{4} B_{2}^{-1}\left[\psi_{1} \cos \left(2 \psi_{s}+2 \psi_{0}\right)+3 \frac{q^{2}}{8} \sin \left(2 \psi_{2}+2 \psi_{0}\right)-3 \frac{q^{2}}{64} \sin \left(4 \psi_{s}+4 \psi_{0}\right)\right]
\end{aligned}
$$

This completes the solution, with exact secular terms and periodic terms correct through order $k^{2}$, for $E, v$, and $\psi$ and thus for the spheroidal coordinates $\rho$ and $\eta$. 


\subsection{The Right Asscension $\phi$}

From (2.7), (5.3), and (6.3) we obtain

$$
\phi=\beta_{3}+\alpha_{3} N_{3}-c^{2} \alpha_{3} R_{3} .
$$

Then, from the equations in sections 5 and 6

$$
\begin{aligned}
\phi=\beta_{3}+\alpha_{3}\left(\alpha_{2}^{2}-\alpha_{3}^{2}\right)^{-\frac{1}{2}} \eta_{0}\left[\left(1-\eta_{0}^{2}\right)^{-\frac{1}{2}}\left(1-\eta_{2}^{-2}\right)^{-\frac{1}{2}} \chi+B_{3} \psi\right. & \\
& \left.+\frac{3}{32} \eta_{0}^{2} \eta_{2}^{-4} \sin 2 \psi\right]-c^{2} \alpha_{3}\left(-2 \alpha_{1}\right)^{-\frac{1}{2}}\left[A_{3} v+\sum_{n=1}^{4} A_{3 n} \sin n v\right],
\end{aligned}
$$

where $\chi$ is an angle that always equals $\psi$ whenever $\psi$ is a multiple of $\pi / 2$ and which also satisfies

$$
\tan \chi=\left(1-\eta_{0}^{2}\right)^{\frac{1}{2}} \tan \psi
$$

The expressions for $A_{3}$ and the $A_{3 n}$ 's are given in (5.61) to (5.65) and that for $B_{3}$ is given in (6.65). With the secular parts of $v$ and $\psi$ exact and their periodic terms correct through order $k^{2}$, the right ascension $\phi$, as given by (8.50), has a secular part that is exact and a periodic part correct through order $k^{2}$. To check the secular part of $\phi$, note that one can obtain it from (8.50) by placing $\chi=\psi=\psi_{s}$ and $v=v_{s}$ and discarding the sines. If we do so and also use $\dot{v}_{s}=2 \pi \nu_{1}$ and $\dot{\psi}_{s}=2 \pi \nu_{2}$, we find

$$
\dot{\phi}_{s}=-2 \pi c^{2} \alpha_{3}\left(-2 \alpha_{1}\right)^{-\frac{1}{2}} A_{3} \nu_{1}+2 \pi \alpha_{3}\left(\alpha_{2}^{2}-\alpha_{3}^{2}\right)^{-\frac{1}{2}} \eta_{0}\left[B_{3}+\left(1-\eta_{0}^{2}\right)^{-\frac{1}{2}}\left(1-\eta_{2}^{-2}\right)^{-\frac{1}{2}}\right] \nu_{2}=-\nu_{1} J_{13}-\nu_{2} J_{23}
$$

on comparison with (7.18) and (7.21). Thus we find

$$
\overline{\dot{\phi}}=\dot{\phi}_{s}=2 \pi \nu_{3}
$$

by (8.51) and (7.14), a result known to be correct [8].

A summary of the principal results of the paper follows in section 9 .

\section{Summary of the Solution}

We assume that $\mu$ and $c$ are known, where $\mu$ is the product of the gravitational constant and the mass of the planet and where $c^{2}=r_{e}^{2} J_{2}, r_{e}$ being the equatorial radius and $J_{2}$ the coefficient of the second zonal harmonic of the planet's gravitational potential. For the earth $J_{2} \approx$ (1.08) $10^{-3}$.

If $X, Y, Z$ are the usual rectangular coordinates of an artificial satellite and if $r, \theta, \phi$ are respectively its planetocentric distance, declination, and right ascension, its oblate spheroidal coordinates $\rho, \eta, \phi$ are given by

The potential

$$
\begin{aligned}
X+i Y & =r \cos \theta \exp i \phi=\left[\left(\rho^{2}+c^{2}\right)\left(1-\eta^{2}\right)\right]^{\frac{1}{2}} \exp i \phi, \\
Z & =r \sin \theta=\rho \eta, \quad(-1 \leqq \eta \leqq 1) .
\end{aligned}
$$

$$
V_{a}=-\mu \rho\left(\rho^{2}+c^{2} \eta^{2}\right)^{-1}
$$

then fits the even zonal harmonics exactly through the second and, in the case of the earth, approximately through the fourth. Solution for the motion with such a potential thus furnishes a very accurate intermediary orbit. Since this potential leads to separability of the HamiltonJacobi equation, the solution is given implicitly by the quadratures of eqs (2.5) through (2.7). The integration constants are the Jacobi $\alpha$ 's and $\beta$ 's.

If the initial conditions are known, one can readily evaluate the $\alpha$ 's. Then if one can evaluate the integrals in (2.5) through $(2.7)$ one can also evaluate the $\beta$ 's. Evaluating the integrals depends on factoring the quartics $F(\rho)$ and $G(\eta)$. The factoring of $G(\eta)$ is immediate, 
since it is quadratic in $\eta^{2}$. To discuss the factoring of $F(\rho)$ we introduce, in place of the $\alpha$ 's, the orbital elements $a_{0} \equiv-\mu / 2 \alpha_{1}, e_{0} \equiv\left[1+2 \alpha_{1} \alpha_{2}^{2} / \mu^{2}\right]^{\frac{1}{2}}$, and $i_{0} \equiv \cos ^{-1}\left(\alpha_{3} / \alpha_{2}\right)$.

With $\rho$ varying in the range $\rho_{1} \leqq \rho \leqq \rho_{2}$, we write

$$
F(\rho) \equiv c^{2} \alpha_{3}^{2}+\left(\rho^{2}+c^{2}\right)\left(-\alpha_{2}^{2}+2 \mu \rho+2 \alpha_{1} \rho^{2}\right)=\left(-2 \alpha_{1}\right)\left(\rho-\rho_{1}\right)\left(\rho_{2}-\rho\right)\left(\rho^{2}+A \rho+B\right)
$$

and find that $A, B, a, p$, and $e$ occur in the $\rho$-integrals.

Here

$$
a \equiv \frac{1}{2}\left(\rho_{1}+\rho_{2}\right), \quad 1-e^{2}=\frac{4 \rho_{1} \rho_{2}}{\left(\rho_{1}+\rho_{2}\right)^{2}}, \quad p \equiv a\left(1-e^{2}\right)=\frac{2 \rho_{1} \rho_{2}}{\rho_{1}+\rho_{2}}, \quad e \equiv \frac{\rho_{2}-\rho_{1}}{\rho_{2}+\rho_{1}}=\left[1-\left(1-e^{2}\right)\right]^{\frac{1}{2}}
$$

Thus $A, B, \rho_{1}+\rho_{2}$, and $\rho_{1} \rho_{2}$ are needed. If we put

$$
p_{0} \equiv a_{0}\left(1-e_{0}^{2}\right), \quad k_{0} \equiv c^{2} / p_{0}^{2}, \quad x \equiv\left(1-e_{0}^{2}\right)^{\frac{1}{2}}, \quad y \equiv \alpha_{3} / \alpha_{2}=\cos i_{0},
$$

then through $O\left(k_{0}^{2}\right)$

$$
\begin{gathered}
A=-2 k_{0} p_{0} y^{2}\left[1+k_{0}\left(2 x^{2}-3 x^{2} y^{2}-4+8 y^{2}\right)+\ldots\right], \\
B=k_{0} p_{0}^{2}\left(1-y^{2}\right)\left[1+k_{0}\left(4 y^{2}-x^{2} y^{2}\right)+\ldots\right], \\
2 a \equiv \rho_{1}+\rho_{2}=2 p_{0} x^{-2}\left[1-k_{0} x^{2} y^{2}-k_{0}^{2} x^{2} y^{2}\left(2 x^{2}-3 x^{2} y^{2}-4+8 y^{2}\right)+\ldots\right], \\
a p \equiv \rho_{1} \rho_{2}=p_{0}^{2} x^{-2}\left[1+k_{0} y^{2}\left(x^{2}-4\right)-k_{0}^{2} y^{2}\left(12 x^{2}-x^{4}-20 x^{2} y^{2}-16+32 y^{2}+x^{4} y^{2}\right)+\ldots\right] .
\end{gathered}
$$

If $a_{0}, e_{0}, i_{0}, \beta_{1}, \beta_{2}, \beta_{3}$ are used as orbital elements, we then assume that the quartic $F(\rho)$ is factored numerically, to as great an accuracy as may be desired, with the aid of the above relations as a starting point.

With $\eta$ varying in the range $-1 \leqq-\eta_{0} \leqq \eta \leqq \eta_{0} \leqq 1$, we write $G(\eta) \equiv-\alpha_{3}^{2}+\left(1-\eta^{2}\right)\left(\alpha_{2}^{2}+2 \alpha_{1} c^{2} \eta^{2}\right)$ $=-2 \alpha_{1} c^{2}\left(\eta_{0}^{2}-\eta^{2}\right)\left(\eta_{2}^{2}-\eta^{2}\right)$ and find

$$
\left(\begin{array}{c}
\eta_{0}^{-2} \\
\eta_{2}^{-2}
\end{array}\right)=\frac{\alpha_{2}^{2}-2 \alpha_{1} c^{2}}{2\left(\alpha_{2}^{2}-\alpha_{3}^{2}\right)}\left[1 \pm\left\{1+\frac{8 \alpha_{1} c^{2}\left(\alpha_{2}^{2}-\alpha_{3}^{2}\right)}{\left(\alpha_{2}^{2}-2 \alpha_{1} c^{2}\right)^{2}}\right\}^{\frac{1}{2}}\right]
$$

Here $\eta_{2} \gg 1$. Then all the quantities $a, p, e, A, B, \eta_{0}$, and $\eta_{2}$ are known in terms of the orbital elements $a_{0}, e_{0}$, and $i_{0}$.

If we assume that the orbital elements are to be determined by an iterated least-square fitting of the solution to many revolutions in the orbit and not by initial conditions, there is a better set of elements, introduced by Izsak [4]. These, viz, $a, e, I \equiv \sin ^{-1} \eta_{0}, \beta_{1}, \beta_{2}$, and $\beta_{3}$, although not easily found from the initial conditions, result in immediate factoring of the quartics $F(\rho)$ and $G(\eta)$. We therefore give the solution in terms of these quantities, with the understanding that they are to be determined either by the least-square fitting or from initial conditions by numerical factoring of $F(\rho)$.

Given $\mu, c$, and the elements $a, e, I, \beta_{1}, \beta_{2}, \beta_{3}$, compute

$$
\begin{aligned}
\eta_{0} & =\sin I, \\
p & =a\left(1-e^{2}\right), \quad D=\left(a p-c^{2}\right)\left(a p-c^{2} \eta_{0}^{2}\right)+4 a^{2} c^{2} \eta_{0}^{2}, \quad D^{\prime}=D+4 a^{2} c^{2}\left(1-\eta_{0}^{2}\right), \\
A & =-2 a c^{2} D^{-1}\left(1-\eta_{0}^{2}\right)\left(a p-c^{2} \eta_{0}^{2}\right), \quad B=c^{2} \eta_{0}^{2} D^{-1} D^{\prime}, \\
b_{1} & =-\frac{1}{2} A, \quad b_{2}=B^{\frac{1}{2}}, \quad-2 \alpha_{1}=\mu\left(a+b_{1}\right)^{-1}, \\
-\frac{\alpha_{2}^{2}}{2 \alpha_{1}} & =a_{0} p_{0}=-c^{2}\left(1-\eta_{0}^{2}\right)+a p D^{-1} D^{\prime}, \quad \alpha_{2}=\left(-2 \alpha_{1}\right)^{\frac{1}{2}}\left(a_{0} p_{0}\right)^{\frac{1}{2}}>0, \\
\alpha_{3} & =\alpha_{2}\left(1-\frac{c^{2} \eta_{0}^{2}}{a_{0} p_{0}}\right)^{\frac{1}{2}} \cos I, \quad \eta_{2}^{-2}=\frac{c^{2} D}{a p D^{\prime}}, \quad k=c^{2} / p^{2}, \quad q=\eta_{0} / \eta_{2} .
\end{aligned}
$$


Restrict considerations to the case $b_{1} / b_{2} \leqq 1$. Then

$$
I_{c} \leqq I \leqq 180^{\circ}-I_{c},
$$

where $I_{c}=1^{\circ} 54^{\prime}$, approximately. Equatorial and almost equatorial orbits are thus ruled out.

Then compute

$$
\begin{aligned}
& A_{1}=\left(1-e^{2}\right)^{\frac{1}{2}} p \sum_{n=2}^{\infty}\left(b_{2} / p\right)^{n} P_{n}\left(b_{1} / b_{2}\right) R_{n-2}\left[\left(1-e^{2}\right)^{\frac{1}{2}}\right], \\
& A_{2}=\left(1-e^{2}\right)^{\frac{1}{2}} p^{-1} \sum_{n=0}^{\infty}\left(b_{2} / p\right)^{n} P_{n}\left(b_{1} / b_{2}\right) R_{n}\left[\left(1-e^{2}\right)^{\frac{1}{2}}\right],
\end{aligned}
$$

where $P_{n}(x)$ is the Legendre polynomial of degree $n$ and where $R_{n}(x) \equiv x^{n} P_{n}\left(x^{-1}\right)$, always a polynomial of degree $[n / 2]$ in $x^{2}$.

where

$$
A_{3}=\left(1-e^{2}\right)^{\frac{1}{2}} p^{-3} \sum_{m=0}^{\infty} D_{m} R_{m+2}\left[\left(1-e^{2}\right)^{\frac{1}{2}}\right]
$$

$$
\begin{gathered}
D_{2 i}=\sum_{n=0}^{i}(-1)^{i-n}(c / p)^{2 i-2 n}\left(b_{2} / p\right)^{2 n} P_{2 n}\left(b_{1} / b_{2}\right), \\
D_{2 i+1}=\sum_{n=0}^{i}(-1)^{i-n}(c / p)^{2 i-2 n}\left(b_{2} / p\right)^{2 n+1} P_{2 n+1}\left(b_{1} / b_{2}\right), \\
B_{1}=2 \pi^{-1} q^{-2}[K(q)-E(q)], \quad B_{2}=2 \pi^{-1} K(q),
\end{gathered}
$$

where $K(q)$ and $E(q)$ are the complete elliptic integrals of the first and second kinds,!respectively.

$$
B_{3}=1-\left(1-\eta_{2}^{-2}\right)^{\frac{1}{2}}-\sum_{m=2}^{\infty} \gamma_{m} \eta_{2}^{-2 m}, \quad \text { where } \quad \gamma_{m} \equiv \frac{(2 m) !}{2^{2 m}(m !)^{2}} \sum_{n=1}^{m-1} \frac{(2 n) ! \eta_{0}^{2 n}}{2^{2 n}(n !)^{2}} .
$$

(The above series all converge rapidly.)

$$
\begin{aligned}
& A_{11}=\frac{3}{4}\left(1-e^{2}\right)^{\frac{1}{2}} p^{-3} e\left(-2 b_{1} b_{2}^{2} p+b_{2}^{4}\right), \quad A_{12}=\frac{3}{32} p^{-3}\left(1-e^{2}\right)^{\frac{1}{2}} b_{2}^{4} e^{2}, \\
& A_{21}=\left(1-e^{2}\right)^{\frac{1}{2}} p^{-1} e\left[b_{1} p^{-1}+\left(3 b_{1}^{2}-b_{2}^{2}\right) p^{-2}-\frac{9}{2} b_{1} b_{2}^{2}\left(1+\frac{e^{2}}{4}\right) p^{-3}+\frac{3}{8} b_{2}^{4}\left(4+3 e^{2}\right) p^{-4}\right] \\
& A_{22}=\left(1-e^{2}\right)^{\frac{1}{2}} p^{-1}\left[\frac{e^{2}}{8}\left(3 b_{1}^{2}-b_{2}^{2}\right) p^{-2}-\frac{9}{8} e^{2} b_{1} b_{2}^{2} p^{-3}+\frac{3}{32} b_{2}^{4}\left(6 e^{2}+e^{4}\right) p^{-4}\right] \\
& A_{23}=\left(1-e^{2}\right)^{\frac{1}{2}} p^{-1} \frac{e^{3}}{8}\left(-b_{1} b_{2}^{2} p^{-3}+b_{2}^{4} p^{-4}\right), \\
& A_{24}=\frac{3}{256}\left(1-e^{2}\right)^{\frac{1}{2}} p^{-5} b_{2}^{4} e^{4}, \\
& A_{31}=\left(1-e^{2}\right)^{\frac{1}{2}} p^{-3} e\left[2+b_{1} p^{-1}\left(3+\frac{3}{4} e^{2}\right)-p^{-2}\left(\frac{1}{2} b_{2}^{2}+c^{2}\right)\left(4+3 e^{2}\right)\right], \\
& A_{32}=\left(1-e^{2}\right)^{\frac{1}{2}} p^{-3}\left[\frac{e^{2}}{4}+\frac{3}{4} b_{1} p^{-1} e^{2}-p^{-2}\left(\frac{e^{4}}{4}+\frac{3}{2} e^{2}\right)\left(\frac{1}{2} b_{2}^{2}+c^{2}\right)\right] \\
& A_{33}=\left(1-e^{2}\right)^{\frac{1}{2}} p^{-3} e^{3}\left[\frac{1}{12} b_{1} p^{-1}-\frac{1}{3} p^{-2}\left(\frac{1}{2} b_{2}^{2}+c^{2}\right)\right] \\
& A_{34}=-\frac{1}{32}\left(1-e^{2}\right)^{\frac{1}{2}} p^{-5} e^{4}\left(\frac{1}{2} b_{2}^{2}+c^{2}\right),
\end{aligned}
$$




$$
\begin{aligned}
& 2 \pi \nu_{1}=\left(-2 \alpha_{1}\right)^{\frac{1}{2}}\left(a+b_{1}+A_{1}+c^{2} \eta_{0}^{2} A_{2} B_{1} B_{2}^{-1}\right)^{-1}, \\
& 2 \pi \nu_{2}=\left(\alpha_{2}^{2}-\alpha_{3}^{2}\right)^{\frac{1}{2}} \eta_{0}^{-1} A_{2} B_{2}^{-1}\left(a+b_{1}+A_{1}+c^{2} \eta_{0}^{2} A_{2} B_{1} B_{2}^{-1}\right)^{-1} .
\end{aligned}
$$

The uniformising variables $E, v$, and $\psi$ are then given by $E=M_{s}+E_{p}, v=M_{s}+v_{p}$, and $\psi=\psi_{s}+\psi_{p}$. If $t$ is the time, their secular parts $M_{s}$ and $\psi_{s}$ are given exactly by

$$
\begin{gathered}
M_{s}=2 \pi \nu_{1}\left(t+\beta_{1}-c^{2} \beta_{2} \alpha_{2}^{-1} \eta_{0}^{2} B_{1} B_{2}^{-1}\right), \\
\psi_{s}=2 \pi \nu_{2}\left[t+\beta_{1}+\beta_{2} \alpha_{2}^{-1}\left(a+b_{1}+A_{1}\right) A_{2}^{-1}\right] .
\end{gathered}
$$

Let the periodic parts be split as follows: $E_{p}=E_{0}+E_{1}+E_{2}, \quad v_{p}=v_{0}+v_{1}+v_{2}$, and $\psi_{p}=\psi_{0}+\psi_{1}+\psi_{2}$, where, e.g., $E_{0}$ contains terms of order $k^{0}, k$, and $k^{2}, E_{1}$ contains terms of order $k$ and $k^{2}$, and $E_{2}$ contains only terms of order $k^{2}$.

Then $E_{0}$ is given by the Kepler equation

$$
M_{s}+E_{0}-e^{\prime} \sin \left(M_{s}+E_{0}\right)=M_{s},
$$

where $e^{\prime} \equiv a\left(a e+b_{1}\right)^{-1}<e$. The term $v_{0}$ is then given by placing $v=M_{s}+v_{0}$ and $E=M_{s}+E_{0}$ in the anomaly connections

$$
\cos v=(\cos E-e)(1-e \cos E)^{-1} \quad \sin v=+\left(1-e^{2}\right)^{\frac{1}{2}}(1-e \cos E)^{-1} \sin E
$$

or equivalent relations. (Note that the $e$ here is the original $e$ and not the $e^{\prime}$ in the Kepler equation.) Then

$$
\psi_{0}=\left(-2 \alpha_{1}\right)^{-\frac{1}{2}}\left(\alpha_{2}^{2}-\alpha_{3}^{2}\right)^{\frac{1}{2}} \eta_{0}^{-1} A_{2} B_{2}^{-1} v_{0} .
$$

The term $E_{1}$ is now given by

where

$$
E_{1}=\left[1-e^{\prime} \cos \left(M_{s}+E_{0}\right)\right]^{-1} M_{1}-\frac{1}{2} e^{\prime}\left[1-e^{\prime} \cos \left(M_{s}+E_{0}\right)\right]^{-3} M_{1}^{2} \sin \left(M_{s}+E_{0}\right),
$$

$$
M_{1} \equiv\left(a+b_{1}\right)^{-1}\left[-\left(A_{1}+c^{2} \eta_{0}^{2} A_{2} B_{1} B_{2}^{-1}\right) v_{0}+\frac{c^{2}}{4}\left(-2 \alpha_{1}\right)^{\frac{1}{2}}\left(\alpha_{2}^{2}-\alpha_{3}^{2}\right)^{-\frac{1}{2}} \eta_{0}^{3} \sin \left(2 \psi_{s}+2 \psi_{0}\right)\right]
$$

The term $v_{1}$ is then given by placing $v=M_{s}+v_{0}+v_{1}$ and $E=M_{s}+E_{0}+E_{1}$ in the anomaly connections. Then

$$
\psi_{1}=\left(-2 \alpha_{1}\right)^{-\frac{1}{2}}\left(\alpha_{2}^{2}-\alpha_{3}^{2}\right)^{\frac{1}{2}} \eta_{0}^{-1} B_{2}^{-1}\left[A_{2} v_{1}+A_{21} \sin \left(M_{s}+v_{0}\right)+A_{22} \sin \left(2 M_{s}+2 v_{0}\right)\right]+\frac{q^{2}}{8} B_{2}^{-1} \sin \left(2 \psi_{s}+2 \psi_{0}\right)
$$

Finally

where

$$
E_{2}=\left[1-e^{\prime} \cos \left(M_{s}+E_{0}+E_{1}\right)\right]^{-1} M_{2},
$$

$$
\begin{aligned}
M_{2} & \equiv-\left(a+b_{1}\right)^{-1}\left[A_{1} v_{1}+A_{11} \sin \left(M_{s}+v_{0}\right)+A_{12} \sin \left(2 M_{s}+2 v_{0}\right)\right. \\
& \left.+c^{2}\left(-2 \alpha_{1}\right)^{\frac{1}{2}}\left(\alpha_{2}^{2}-\alpha_{3}^{2}\right)^{-\frac{1}{2}} \eta_{0}^{3}\left\{B_{1} \psi_{1}-\frac{1}{2} \psi_{1} \cos \left(2 \psi_{s}+2 \psi_{0}\right)-\frac{q^{2}}{8} \sin \left(2 \psi_{s}+2 \psi_{0}\right)+\frac{q^{2}}{64} \sin \left(4 \psi_{s}+4 \psi_{0}\right)\right\}\right] .
\end{aligned}
$$

Then $v_{2}$ is found by placing $v=M_{s}+v_{0}+v_{1}+v_{2}$ and $E=M_{s}+E_{0}+E_{1}+E_{2}$ in the anomaly connections and

$$
\begin{aligned}
\psi_{2}=\left(-2 \alpha_{1}\right)^{-\frac{1}{2}}\left(\alpha_{2}^{2}-\alpha_{3}^{2}\right)^{\frac{1}{2}} \eta_{0}^{-1} B_{2}^{-1}[ & A_{2} v_{2}+A_{21} v_{1} \cos \left(M_{s}+v_{0}\right) \\
+2 A_{22} v_{1} \cos ( & \left.\left.2 M_{s}+2 v_{0}\right)+A_{23} \sin \left(3 M_{s}+3 v_{0}\right)+A_{24} \sin \left(4 M_{s}+4 v_{0}\right)\right] \\
+ & \frac{q^{2}}{4} B_{2}^{-1}\left[\psi_{1} \cos \left(2 \psi_{s}+2 \psi_{0}\right)+\frac{3 q^{2}}{8} \sin \left(2 \psi_{s}+2 \psi_{0}\right)-\frac{3 q^{2}}{64} \sin \left(4 \psi_{s}+4 \psi_{0}\right)\right] .
\end{aligned}
$$

The spheroidal coordinates $\rho$ and $\eta$ are then given by

$$
\begin{aligned}
& \rho=a(1-e \cos E)=(1+e \cos v)^{-1} p, \quad \eta=\eta_{0} \sin \psi, \text { where } \\
& E=M_{s}+E_{0}+E_{1}+E_{2}, \quad v=M_{s}+v_{0}+v_{1}+v_{2}, \quad \psi=\psi_{s}+\psi_{0}+\psi_{1}+\psi_{2} .
\end{aligned}
$$


The right ascension is

$$
\begin{array}{r}
\phi=\beta_{3}+\alpha_{3}\left(\alpha_{2}^{2}-\alpha_{3}^{2}\right)^{-\frac{1}{2}} \eta_{0}\left[\left(1-\eta_{0}^{2}\right)^{-\frac{1}{2}}\left(1-\eta_{2}^{-2}\right)^{-1 / 2} \chi+B_{3} \psi+\frac{3}{32} \eta_{0}^{2} \eta_{2}^{-4} \sin 2 \psi\right] \\
\quad-c^{2} \alpha_{3}\left(-2 \alpha_{1}\right)^{-\frac{1}{2}}\left[A_{3} v+\sum_{n=1}^{4} A_{3 n} \sin n v\right] .
\end{array}
$$

Here $\chi$ is an angle that equals $\psi$ whenever $\psi$ is a multiple of $\pi / 2$ and which also satisfies $\tan \chi=\left(1-\eta_{0}^{2}\right)^{\frac{1}{2}} \tan \psi$. If $\alpha_{3}$ is positive or negative, the orbit is respectively direct or retrograde.

The above solution gives secular terms of the intermediary orbit exactly and periodic terms correctly through order $k^{2}$. There are no long-period terms.

\section{List of Symbols}

We here list only the symbols that are most frequently used, giving for each a short definition or the number of the equation in which it first appears. Note that the first digit in each such number is the number of the section.
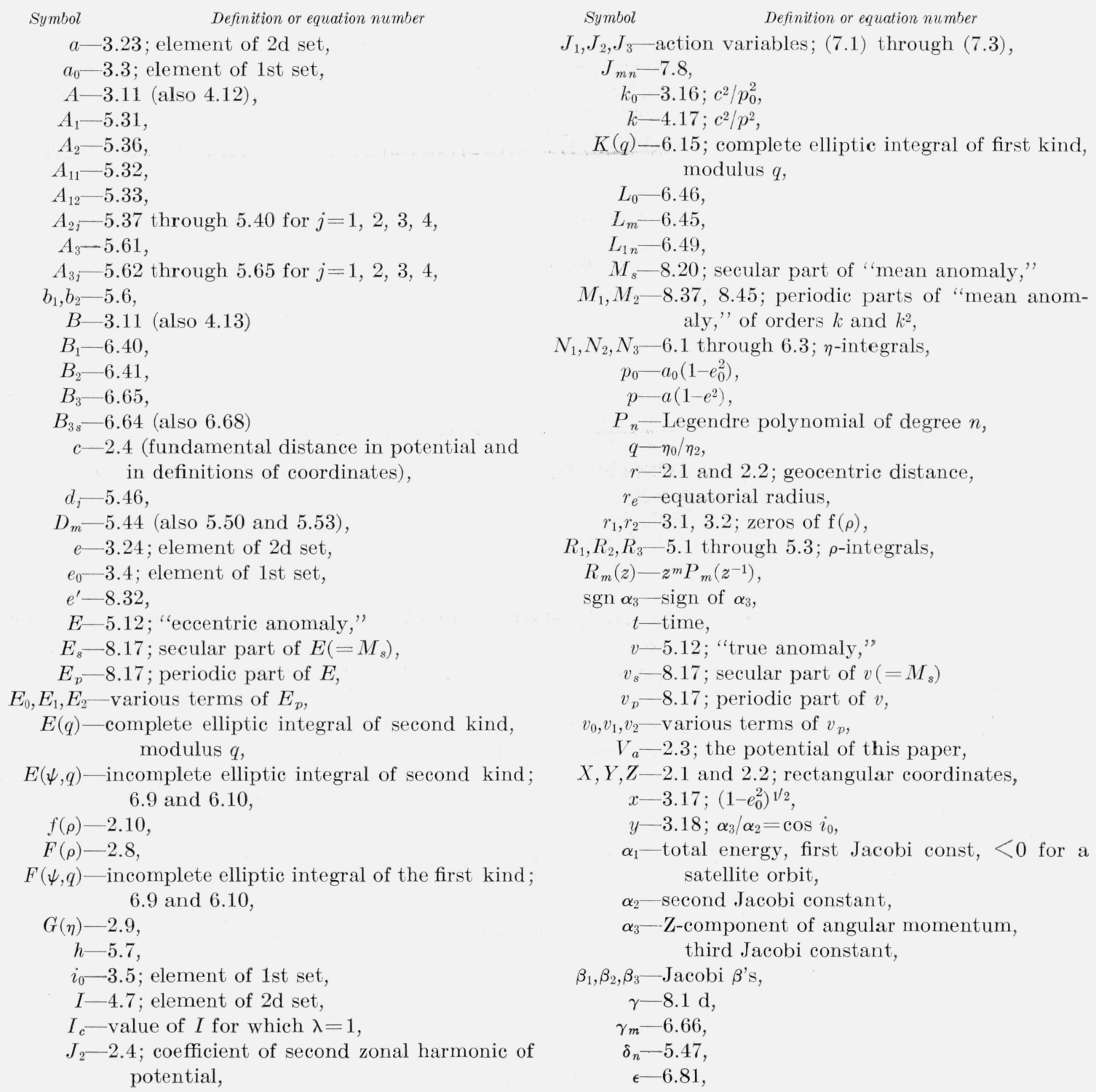
$\eta-2.1$ and 2.2 ; a spheroidal coordinate $\rightarrow \sin \theta$ as $r \rightarrow \infty$,

$\eta_{0}-3.33,3.36,3.39$; during motion

$-1 \leqq-\eta_{0} \leqq \eta \leqq \eta_{0} \leqq 1$,

$\eta_{2}-3.33,3.37, \eta_{2}>>1$,

$\theta-2.1$ and 2.2 ; geocentric declination,

$\lambda-5.6 ; b_{1} / b_{2}$,

$\boldsymbol{\nu}_{1}, \nu_{2}, \nu_{3}-7.4$ through 7.6 ,

$\rho-2.1$ and 2.2 ; a spheroidal coordinate $\rightarrow r$ as $r \rightarrow \infty$,

$\phi-2.1$ and 2.2 ; geocentric right ascension; the third spheroidal coordinate, $\chi-6.51$,

$\psi-6.4$; a uniformizing variable analogous to the argument of latitude,

$\psi_{s}-8.17$; secular part of $\psi$,

$\psi_{D}-8.17$; periodic part of $\psi$, $\psi_{0}, \psi_{1}, \psi_{2}$-various terms of $\psi_{p}$,

$\overline{\dot{\omega}}$-mean motion of $\rho$-perigee relative to the ascending node,

$\overline{\dot{\Omega}}$ - mean motion of the ascending node relative to $O X$.

\section{References}

[1] J. P. Vinti, J. Research NBS 63B, 105-116 (1959).

[2] T. E. Sterne, Astron. J. 62, 96 (1957); 63, 28-40 (1958).

[3] B. Garfinkel, Astron. J. 63, 88-96 (1958); 64, 353-367 (1959).

[4] I. Izsak, Smithsonian Institution Astrophysical Observatory, Research in Space Science, Special Rept. No. 52, (1960).

[5] E. T. Whittaker and G. N. Watson, Modern Analysis, 4th ed., p. 312 (Cambridge Univ. Press, Cambridge, 1952).

[6] E. T. Whittaker and G. N. Watson, Modern Analysis, 4th ed., p. 29 (Cambridge Univ. Press, Cambridge, $1952)$.

[7] E. T. Whittaker and G. N. Watson, Modern Analysis, 4th ed., p. 49 (Cambridge Univ. Press, Cambridge, 1952).

[8] J. P. Vinti, J. Research NBS 65B, 131-135 (1961).

(Paper 65B3-56) 